\title{
COMPLETE TIME-REVERSED REFOCUSING IN REFLECTION WITH AN ACOUSTIC LAGRANGIAN MODEL*
}

\author{
DANIEL G. ALFARO VIGO ${ }^{\dagger}$, ADOLFO G. S. CORREIA ${ }^{\ddagger}$ AND ANDRÉ NACHBIN $§$
}

\begin{abstract}
Discrete reflection-transmission acoustic models are introduced and analysed regarding their underlying physical properties. Namely, phenomena related to multiple scattering as encountered in the underlying continuous model. Moreover, the discrete models are designed so that computational experiments can be performed efficiently. Each discrete model is expressed through its corresponding reflection-transmission matrix establishing a connection with lattice models encountered in the Physics literature. Their connections with the continuous acoustic model are discussed in detail. In particular we show how a Goupillaud medium can arise from a stable discretization of a more general random medium. Related physical phenomena are studied computationally. In particular reflection-transmission properties of waves in a rapidly varying random medim, which are valid over long propagation distances. By using a long computational domain, in a regime where we have Anderson localization, the energy of an incoherently scattered signal is entirely reflected back and, by time-reversal, completely recompressed into the smooth initial data. Experiments are also performed in a regime where separation of scales fails to hold. Another new result is the time-reversed refocusing, in reflection, of a wave train in the form of a bit stream. The bit stream is scrambled by reflection and unscrambled through time reversal. The robustness of the time-reversed refocusing phenomenon is outstanding.
\end{abstract}

Key words. Inhomogeneous media, asymptotic theory, time reversal

AMS subject classifications. 35L05, 60H15, 74J20

\section{Introduction}

Time-reversal techniques have emerged as a new important and fascinating discipline within wave propagation in heterogeneous media $[20,19]$. They are based on the use of a time-reversal mirror, which is a device capable of receiving a signal in time, keeping it in memory and re-emitting it back into the medium in the reversed direction of time. The main effect is the refocusing of the scattered signal after time-reversal in a random medium: an acoustic pulse is sent in a disordered medium generating a highly fluctuating, scattered signal which is time-reversed and sent back into the medium. The new scattered signal is a pure pulse with a shape similar to the initial pulse. Amazingly its "refocusing" takes place in time and space and seems to be independent of the realization of the medium. The pulse shape is of a deterministic character. This is called the stabilization of the refocused pulse shape and is due to the self-averaging property [12]. Furthermore, in some situations the randomness of the medium helps to improve refocusing "beating" the diffraction limit $[7,6,15]$. Potential applications of these effects are numerous in imaging, wireless communication, medicine, geophysics, underwater acoustic, among others as found in $[35,27,28,15,26]$.

Several regimes and scalings can be adopted for studying this fascinating phenomenon. We chose to work in the regime where the medium's correlation length is smaller than the typical wavelength of the pulse. This choice has been made

\footnotetext{
*Received: May 17, 2006; accepted (in revised version): December 25, 2006. Communicated by Lenya Ryzhik.

${ }^{\dagger}$ Department of Mathematics, University of California, Irvine, CA 92697, USA (dgalfaro@math. uci.edu).

${ }^{\ddagger}$ Instituto Militar de Engenharia, Praça Gen. Tibúrcio 80, Rio de Janeiro, RJ 22290-270, Brazil.

§Instituto de Matemática Pura e Aplicada, Est. D. Castorina 110, Jardim Botânico, Rio de Janeiro, RJ 22460-320, Brazil (nachbin@impa.br).
} 
due to a great amount of well-founded mathematical theory (in the corresponding wave/medium regime) that can be found, for example, in $[4,9,10]$ and the references within. Nevertheless it is well known that this is not the only regime for time-reversed refocusing to take place. Results in the high-frequency regime (where the correlation length is comparable to the carrier wavelength) can be found in $[5,7,13,14,21]$.

From the theoretical point of view, a first proof of this refocusing effect has been obtained by Clouet and Fouque [11] in the context of a one-dimensional random medium for which only the time refocusing is relevant. The refocusing is obtained by using asymptotics in the regime where the incident pulse propagates a long distance in comparison to its characteristic length through a medium that fluctuates on a fine scale relative to the pulse width. The fluctuations of the medium are not assumed to be small.

The aim of this paper is to analyse discrete reflection-transmission acoustic models through which we can perform efficient and robust numerical experiments. These experiments are intended to assist in both theoretical and experimental investigations. Hence we are interested in providing evidence that important physical properties are retained by the discrete model. In particular important properties related to the refocusing phenomenon in time-reversal.

We focus our experiments on the time-reversal in reflection (TRR) regime, which has been much less explored than time-reversal in transmission (TRT) $[5,7,13,14$, $20,21,28]$.

Anderson localization [2] is a well-known phenomenon for linear waves in a onedimensional (1D) random medium and has been mathematically analyzed for quite some time [3, 24]. Nevertheless, as will be pointed out in more detail later on, many recent papers $[32,16]$ consider the study of Anderson localization in lattices, graphs and other discrete systems. By using a long computational domain, in a regime/scaling where we have Anderson localization [3], we expect that the entire energy of the initial data is backscattered as an incoherent signal. Namely, no transmission is (effectively) observed over the (effective) bandwidth of the propagating pulse. Time reversing the long incoherent (reflected) signal gives rise to a smooth pulse identical to the initial profile. Our goal is to reproduce this known result with our discrete model: the incoherent signal is completely recompressed into a smooth pulse, hence illustrating the stochastic result given by Clouet and Fouque [11]. Moreover, in a subsequent experiment we violate the separation of scales hypothesis used in [11] by considering a smooth varying background. Experiments with the discrete model show the robustness of time reversal refocusing.

Another new result is the time-reversed refocusing, in reflection, of a wave train in the form of a bit stream. Our main goal is to show how the bit stream can be scrambled by back scattering and accurately unscrambled by time reversal. In this paper transmission is not an issue as for example in a wireless communication applications where many recent studies have considered time-reversal as a robust tool for sending and encoding bit streams $[15,26,27]$. The robustness of the time-reversed refocusing phenomenon is outstanding. Robustness had already been analyzed in a different context, where the random medium changes slightly in between the forward and the time-reversed experiment. The corresponding stochastic theory and numerical results are presented in Alfaro et al. [1]. The numerical experiments were performed with the discrete model described (in detail, for the first time) in the present paper. The agreement between the theoretical expressions and numerical results were excellent.

Recently Sølna [33] presented interesting numerical results for TRR, in the high 
frequency regime, for an impulsive source interacting with weak fluctuations in a Goupillaud medium. In contrast, we time-reverse the incoherent backscattered signal generated by a broad pulse interacting with a strongly fluctuating medium. Our medium is not composed of slabs of equal traveltime (as in a Goupillaud medium; c.f. appendix A). Another interesting numerical work is given by [25] in which again the Goupillaud structure is taken advantage of numerically by using the Green's function over each subinterval. The evolution is done in frequency space (through a frequency dependent transfer matrix) and a boundary integral formulation leads to an efficient banded system to be solved numerically. In contrast our discrete lagrangian model leads to transfer (reflection-transmission) matrices in physical space.

This paper is organized as follows, in Section 2 we present the model of a continuous one-dimensional acoustic medium, and for completeness in the appendix A a commonly used discrete model, i.e. a Goupillaud layered medium. In Section 3 we briefly review the asymptotic theory of time-reversal refocusing [11]. In Section 4 the discretization of the equations is carried out, remarking the relationship with the Goupillaud layered medium (appendix A) and setting up a numerical framework for experiments. Additionally, in appendix B we prove that this discrete model represents a good approximation of its continuous counterpart. Finally, in Section 5 we present some numerical experiments illustrating time-reversal refocusing and the relevant physical properties of the discrete model.

\section{One-dimensional acoustic waves}

The governing equations for wave propagation through a one-dimensional acoustic medium are the conservation laws for momentum and mass:

$$
\begin{aligned}
\varrho \partial_{t} u+\partial_{z} p & =0 \\
\frac{1}{\kappa} \partial_{t} p+\partial_{z} u & =0,
\end{aligned}
$$

where $p(z, t)$ is the pressure and $u(z, t)$ the velocity. The medium coefficients $\varrho(z)$ and $\kappa(z)$ are, respectively, the density and bulk modulus, and the acoustic sound speed is given by

$$
c(z)=\sqrt{\frac{\kappa(z)}{\varrho(z)}} .
$$

The characteristics curves are defined as solutions of the ordinary differential equations $\mathrm{d} z / \mathrm{d} t= \pm c(z)$, which are highly oscillatory when the medium properties vary rapidly in space. By introducing (as a new 'space-related' coordinate) the travel time from the origin

$$
x=\int_{0}^{z} \frac{\mathrm{d} s}{c(s)},
$$

system (2.1) can be put in the form

$$
\begin{aligned}
& \zeta \partial_{t} u+\partial_{x} p=0 \\
& \partial_{t} p+\zeta \partial_{x} u=0
\end{aligned}
$$

where $\zeta$ is the acoustic impedance of the medium defined as

$$
\zeta(x)=\sqrt{\varrho(x) \kappa(x)} .
$$


We remark that due to the change of variable (2.3) system (2.4) has a family of characteristic lines

$$
C_{\alpha}^{ \pm}:\left\{x=x^{ \pm}(t)= \pm t+\alpha\right\}, \quad \alpha \in \mathbb{R}
$$

as opposed to the highly oscillatory characteristics of system (2.1). Moreover in the homogeneous case, with $\zeta$ constant, it has the Riemann invariants

$$
\begin{aligned}
& D=\zeta^{\frac{1}{2}} u+\zeta^{-\frac{1}{2}} p \\
& U=-\zeta^{\frac{1}{2}} u+\zeta^{-\frac{1}{2}} p
\end{aligned}
$$

representing right- and left-going waves respectively. By applying the operators $\partial_{t} \pm$ $\partial_{x}$ to (2.7) and using (2.4) we obtain

$$
\begin{aligned}
& \left(\partial_{t}+\partial_{x}\right) D=-r U \\
& \left(\partial_{t}-\partial_{x}\right) U=r D
\end{aligned}
$$

with the reflectivity coefficient

$$
r(x)=\frac{\zeta^{\prime}}{2 \zeta}=\frac{1}{2}(\log \zeta)^{\prime}=\frac{\left(\zeta^{1 / 2}\right)^{\prime}}{\zeta^{1 / 2}} .
$$

We clearly see that in a homogeneous medium $D$ is a traveling wave to the right and $U$ to the left, and that they are completely uncoupled. The coupling of the Riemann invariants is through the reflectivity, in the forcing term. The multiple scattering nature of this model is evident through the constant exchange of energy between the left and right propagating modes.

Conservation of mechanical energy is another important feature concerning acoustic wave propagation. For solutions of (2.4) it takes the form

$$
E(t)=\frac{1}{2} \int_{-\infty}^{+\infty}\left(\rho u^{2}+\frac{1}{\kappa} p^{2}\right) \mathrm{d} z=\text { constant }
$$

and according to the transformations introduced above, in the new variables, the following identity holds:

$$
E(t)=\frac{1}{4} \int_{-\infty}^{+\infty}\left(D^{2}+U^{2}\right) \mathrm{d} x=\text { constant }
$$

In the next section we will discuss a situation where the leading front of the transmitted wave seems to be under a diffusive-like behavior. This is an apparent diffusion since the energy being lost (in a diffusive-like manner) by, say, mode $D$ is being stored as incoherent energy in mode $U$. The total energy is being conserved as expressed through the conservation law (2.11).

\section{Time-reversed refocusing in reflection}

This section contains a brief review of the mathematical results given by Clouet and Fouque [11] on time-reversal in reflection. As will be noticed below, the recorded signal is formulated essentially the same way as found in the literature as, for example, in $[11,12]$.

We discuss the regime of separation of scales in which the ratio of the correlation length of the medium fluctuations to typical pulse wavelength is small and, comparable 
$\mathrm{z}=0$

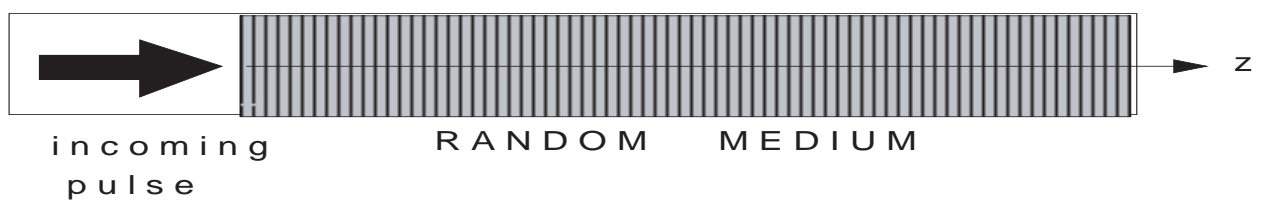

FIG. 3.1. A schematic representation of the random medium.

to the ratio of the typical wavelength to propagation distance. The medium considered is a random half-space with its corresponding properties modeled through the variable coefficients as

$$
\begin{gathered}
\varrho(z)=\left\{\begin{array}{cc}
\varrho_{0}\left(1+\eta\left(\frac{z}{\varepsilon}\right)\right), & \text { for } z \geq 0 \\
\varrho_{0}, & \text { for } z<0
\end{array}\right. \\
\frac{1}{\kappa(z)}=\left\{\begin{array}{cc}
\frac{1}{\kappa_{0}}\left(1+\nu\left(\frac{z}{\varepsilon}\right)\right), & \text { for } z \geq 0 \\
\frac{1}{\kappa_{0}}, & \text { for } z<0 .
\end{array}\right.
\end{gathered}
$$

Here the background properties, expressed through $\varrho_{0}$ and $\kappa_{0}$, are constant. A schematic picture is presented in Fig. 3.1. The theory [11, 22] allows for slow background variations but for simplicity we present our overview in this simpler scenario. In one set of numerical experiments we will consider background variations. First having the background varying on a slow scale, as determined by the theory, and then on a faster scale violating the separation of scales hypothesis. We will see that time-reversed refocusing is quite robust.

The fluctuations are determined through the centered stationary random processes $\eta(\cdot), \nu(\cdot)$, which are bounded by a deterministic constant (i.e. $|\eta|<C,|\nu|<C$, almost surely with $C<1)$. They are considered exponentially mixing in the sense that (each) decorrelates exponentially fast, and have a correlation length of $O(1)$. The above scaling assures that the random fluctuations have correlation length of $O(\varepsilon)$, and we assume that $\varepsilon>0$ is a small parameter. Under this scaling the heterogeneous medium is rapidly varying with respect to the propagating pulse of $O(1)$ width. We remark that we do not consider the fluctuations to be small.

In the homogeneous half-space $z<0$ the Riemann invariants $D, U$ correspond to right- and left-going waves. Thus in that region the original wave fields are easily obtained through

$$
\begin{aligned}
& u(z, t)=\frac{D_{0}\left(t-z / c_{0}\right)-U_{0}\left(t+z / c_{0}\right)}{2 \zeta_{0}^{1 / 2}} \\
& p(z, t)=\frac{\zeta_{0}^{1 / 2}}{2}\left(D_{0}\left(t-z / c_{0}\right)+U_{0}\left(t+z / c_{0}\right)\right),
\end{aligned}
$$

where $c_{0}=\sqrt{\kappa_{0} / \varrho_{0}}, \zeta_{0}=\sqrt{\varrho_{0} \kappa_{0}}$ are the sound speed and acoustic impedance in the homogeneous half-space. We have used the notation $D_{0}(t)=D(x=0, t), U_{0}(t)=U(x=$ $0, t)$. Consequently, for a pulse impinging upon the heterogeneous half-space from the left we have, at the interface, the reflected signals

$$
u^{\mathrm{ref}}(0, t)=-\frac{U_{0}(t)}{2 \zeta_{0}^{1 / 2}}, \quad p^{\mathrm{ref}}(0, t)=\frac{\zeta_{0}^{1 / 2} U_{0}(t)}{2} .
$$


If we consider a pulse with typical wavelength of $O(1)$ and observe its evolution for a relatively small time, since the correlation length of the fluctuations (3.1) is of $O(\varepsilon)$, one sees according to homogenization theory that the pulse practically does not generate reflection and travels with velocity $c_{0}$ without changing its shape [3]. However, if we continue observing for a longer time, reflections appear and the pulse moves with a random speed and changes its shape, in a deterministic fashion, according to the O'Doherty-Anstey (ODA) approximation (see [3, 22]). To leading order the deterministic pulse shaping is diffusive-like. In accordance with these remarks, the time-reversal procedure should be implemented for a sufficiently large time intervals.

The time-reversal procedure goes as follows: (i) let a right-going pulse impinge upon the heterogeneous half-space, (ii) record the reflected time signal at the interface up to a time $t_{0}$ and (iii) reverse its time direction. Hence this incoherent signal is used as new initial data being re-emitted backwards into the medium. A new reflected signal is generated at the interface and its subsequent refocusing can be stated as follows: if we observe this time-reversed reflection for a sufficiently long time $\left(t>t_{0}\right)$ then a left-going pulse, that resembles the initial one, emerges at the interface exactly at $t=t_{0}$ and continues to travel to the left, into the homogeneous medium. This will be shown in the numerical experiments.

Mathematically, this property is established through an asymptotic analysis (as $\varepsilon \downarrow 0)$ for the random differential equations which model the pulse reflection process (for details see [11, 22]). In this analysis the scaling is crucial for establishing the diffusion approximation that allows for the use of the powerful tools of stochastic calculus.

More precisely, we consider that the recording and observation times are long and satisfy $t_{0}=\varepsilon^{-1} t_{0}^{\prime}$ and $t_{1}=\varepsilon^{-1} t_{1}^{\prime}$, respectively. A cutoff function $G_{t_{0}}(t)$, supported on the interval $\left[0, t_{0}\right]$ represents (mathematically) the recording process. It is considered to vary slowly, in the sense that

$$
G_{t_{0}}(t)=G_{t_{0}^{\prime}}(\varepsilon t)
$$

where $G_{t_{0}^{\prime}}$ is smooth in $\left(0, t_{0}^{\prime}\right)$. In the simplest situation, one has $G_{t_{0}}=\mathbf{1}_{\left[0, t_{0}\right]}$ the indicator function for the interval $\left[0, t_{0}\right]$.

The refocusing property is stated as follows. For an initial right-going pulse $D_{0}(t)=f(t)$ the limiting (as $\left.\varepsilon \downarrow 0\right)$ time-reversed reflection observed at the time $t_{1}$ satisfies [11]:

$$
\lim _{\varepsilon \downarrow 0} U_{0}^{\varepsilon, \mathrm{TR}}\left(\frac{t_{1}^{\prime}}{\varepsilon}+t\right)=\left\{\begin{array}{cc}
f_{G_{t_{0}^{\prime}}}^{\mathrm{TR}}(t), & \text { for } t_{1}^{\prime}=t_{0}^{\prime} \\
0, & \text { for } t_{1}^{\prime} \neq t_{0}^{\prime}
\end{array}\right.
$$

where the refocused time-reversed (TR) pulse is

$$
f_{G_{t_{0}^{\prime}}^{\prime}}^{\mathrm{TR}}(t)=\left(H_{G_{t_{0}^{\prime}}}(\cdot) \star f(-\cdot)\right)(t)
$$

with the (limiting) refocusing kernel $H_{G_{t_{0}^{\prime}}}(\cdot)$ given by

$$
\hat{H}_{G_{t_{0}^{\prime}}}(\omega)=\left(\Lambda(\omega, \cdot) \star G_{t_{0}^{\prime}}(-\cdot)\right)(0) .
$$

By $\hat{g}$ we represent the Fourier transform of the function $g$ and the symbol $\star$ stands for convolution with respect to $t$. The function $\Lambda(\omega, t)$ is explicitly given by (see $[9,22]$ )

$$
\Lambda(\omega, t)=\frac{\omega^{2} \gamma_{n}}{\left(1+\omega^{2} \gamma_{n} t\right)^{2}}
$$


with

$$
\gamma_{n}=\frac{1}{c_{0}} \int_{0}^{\infty} \mathbb{E}\{n(s) n(0)\} \mathrm{d} s,
$$

and $n(s)=(\eta(s)-\nu(s)) / 2$ (c.f. (3.1)). It is the limiting power spectral density associated with the random signal scattered at the interface $[3,9,10]$.

A remarkable point is that the limiting form for the (time-reversed) refocused pulse is deterministic and depends only on one statistical property of the random medium as indicated in (3.7). This result is known as pulse stabilization (see $[11,12$, 22]). Actually, the limit in (3.3) expresses convergence in probability. Consequently, the observed refocused pulse stays arbitrarily close to the deterministic signal (3.4) with a probability that approaches 1 as $\varepsilon \downarrow 0$.

As mentioned above, a better understanding on the shape of the refocused pulse is achieved by analysing the case of a time-reversal mirror, with $G_{t_{0}^{\prime}}=\mathbf{1}_{\left[0, t_{0}^{\prime}\right]}$. We simplify the kernel's notation by using the subscript $t_{0}^{\prime}$ in place of $G_{t_{0}^{\prime}}$ in the representation of the corresponding refocused pulse and refocusing kernel. In this particular case we get that the Fourier transform of the refocusing kernel $H_{t_{0}^{\prime}}$ is given by

$$
\hat{H}_{t_{0}^{\prime}}(\omega)=\frac{\omega^{2} \gamma_{n} t_{0}^{\prime}}{1+\omega^{2} \gamma_{n} t_{0}^{\prime}} .
$$

Therefore $H_{t_{0}^{\prime}}$ is a highpass filter, such that when $t_{0}^{\prime}$ goes to infinity $H_{t_{0}^{\prime}}$ tends to a Dirac $\delta$-function. Moreover, we have the following complete refocusing property. There exists a real constant $c_{1}>0$ such that for any $t_{0}^{\prime}>0$

$$
\lim _{\varepsilon \downarrow 0} \mathbb{P}\left\{\left\|U_{0}^{\varepsilon, \mathrm{TR}}\left(\frac{t_{0}^{\prime}}{\varepsilon}+\cdot\right)-f(-\cdot)\right\|_{\infty}<c_{1}\|\hat{f}\|_{\infty}\left(\gamma_{n} t_{0}^{\prime}\right)^{-1 / 2}\right\}=1,
$$

where $\|g\|_{\infty}=\sup _{t \in \mathbb{R}}|g(t)|$ represents the usual $L^{\infty}$-norm of $g(t)$. So we have that as the recording time grows, the limiting refocused pulse shape approaches the initial pulse waveform. Hence the original pulse is fully recovered through time-reversal in reflection. The long incoherent reflected signal recompresses into the smooth initial profile. This has never been shown numerically and is reminiscent of Anderson localization [2]. Namely, the random medium is opaque (to all frequencies) and there is no transmission. All the energy is reflected back and by time-reversed recompression the initial pulse is fully recovered. Moreover, for the limiting time-reversed reflection, after some elementary estimates we get that if $\left\|G_{t_{0}^{\prime}}\left(t^{\prime}\right)\right\|_{\infty} \leq 1$ then

$$
\left\|f_{G_{t_{0}^{\prime}}}^{\mathrm{TR}}\right\|_{2} \leq\left\|f_{t_{0}^{\prime}}^{\mathrm{TR}}\right\|_{2}
$$

where $\|g\|_{2}=\left(\int|g(t)|^{2} \mathrm{~d} t\right)^{1 / 2}$ represents the $L^{2}$-norm of the time-signal $g(t)$. Furthermore, this is a sharp estimate and if additionally we only consider non-negative cutoff functions then the equality holds if and only if $G_{t_{0}^{\prime}}=\mathbf{1}_{t_{0}^{\prime}}$. Hence the uniform cutoff function is the best for energy recovery through recompression.

\section{Discrete reflection-transmission systems}

In this section we analyse discrete reflection-transmission systems which can be used, for example, in numerical experiments. We present two ways of obtaining the discrete physical models. First, we use numerical methods for approximating the system of partial differential equations. We show that in one particular case we obtain a Goupillaud medium commonly used in Geophysics. Both finite difference 
schemes presented have a discrete analog for the energy conservation law. We also study a discrete physical model by approximating evolution operators.

Our main goal in this paper is to provide strong evidence that the underlying discrete models retain some relevant physical properties of the continuum model. We establish this by carrying out a convergence analysis in appendix B and through the numerical simulations presented in section 5. This is interesting from the lattice viewpoint and also as a computational tool that can assist laboratory experiments.

In the recent Physics literature one can find interesting research articles concerning reflection-transmission on lattices and graphs, all including the study of 1D Anderson localization. For example in Schanz and Smilanskyi [32] (equations (1) and (2)) a scattering matrix formulation is used. This formulation is basically identical to ours, given below. Schanz and Smilanskyi study localization in a 1D chain topology. The matching conditions across the graph's vertex is given by a unitary scattering matrix. The transmission and reflection coefficients are given at the bottom of page 1427 [32] followed by a unitary operator that describes the time evolution map. This is the essence of our presentation for the discrete models given below. We called this section "Discrete reflection-transmission system" exactly to establish the connection with, say, chains, lattices, graphs and other discrete models. In an even more recent paper, Dominguez-Adame and Malyshev [16], also study 1D Anderson localization, but on a disordered lattice where random energy are assigned to each lattice site. This role is played in our paper by the random reflectivity assigned at each node.

It is interesting to note that in both references $[32,16]$ the analysis is performed in wavenumber space. In the following subsections we will show that through the use of Riemann invariants we get the scattering matrices, and develop discrete/lattice models, for a full band of wavenumbers, directly in physical space.

We shall develop our discrete models related to (2.1), by basing our analysis on the equivalent equations (2.8) that are more suitable for the study of the right- and leftgoing waves, and therefore for reflection-transmission problems. As mentioned earlier, the velocity and pressure fields can be easily obtained by using transformation (2.7). The change of variable (2.3) can be carried out accurately by solving the corresponding initial value problem (in $z$ ) through a suitable method. For simplicity we consider that this coordinate change has been performed exactly.

We point out that the random medium under consideration does not need to be piecewise constant. Thus we obtain reflection-transmission matrices corresponding to the discrete scattering process for quite general random media profiles. We have to stress that all discrete schemes presented conserve energy and are convergent. Moreover the implicit schemes developed are such that there is no need to solve an algebraic system of equations.

4.1. Semi-lagrangian schemes. The semi-lagrangian approach in the computation of highly oscillatory solutions to hyperbolic differential equations has been successfully used. An important reason for this, is its ability to perform the correct advection of high frequency modes, eliminating one of the central source of problems in the discrete approximations of the equations $[17,18]$. We shall follow this approach in the discretization of (2.8) to obtain an implicit conservative scheme.

We consider the spatio-temporal grid $\left\{\left(x_{j}, t_{n}\right), j \in \mathbb{Z}, n \in \mathbb{Z}_{+}\right\}$with $\Delta x=\Delta t=h$ and use the representation

$$
\begin{aligned}
& D_{j}^{n}=D\left(x_{j}, t_{n}\right) \\
& U_{j}^{n}=U\left(x_{j}, t_{n}\right)
\end{aligned}
$$


where $\left(x_{j}, t_{n}\right)=(j h, n h)$.

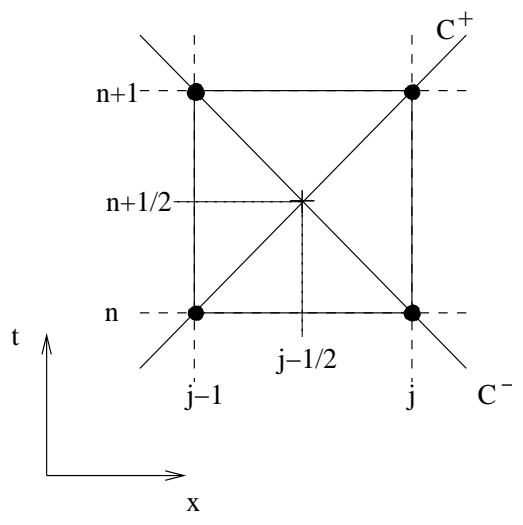

FIG. 4.1. Characteristic lines, spatio-temporal grid and staggered gridpoints.

We shall approximate equation (2.8) in the staggered gridpoints $\left(x_{j-1 / 2}, t_{n+1 / 2}\right)$ (see Fig. 4.1). The differential operators $\partial_{t} \pm \partial_{x}$ are discretized by considering them as directional derivatives along the characteristics $C^{ \pm}$using a central difference approximation. The right-hand side $D, U$ are approximated by a midpoint averaging on the characteristics $C^{ \pm}$, respectively, and the reflectivity coefficient (2.9) using a finite difference approximation supported on the spatial grid points. We obtain

$$
\begin{aligned}
\frac{D_{j}^{n+1}-D_{j-1}^{n}}{h} & =-\tilde{r}_{j-1 / 2}\left(\frac{U_{j-1}^{n+1}+U_{j}^{n}}{2}\right) \\
\frac{U_{j-1}^{n+1}-U_{j}^{n}}{h} & =\tilde{r}_{j-1 / 2}\left(\frac{D_{j}^{n+1}+D_{j-1}^{n}}{2}\right)
\end{aligned}
$$

where $\tilde{r}_{j-1 / 2}$ represents the approximation of the reflectivity coefficient. There are different ways to approximate the reflectivity, which gives place to different discrete reflection-transmission models. In particular we will show that one of our choices falls exactly on the Goupillaud medium commonly used by geophysicists in seismic analysis.

For instance, we can use one of the following approximations:

$$
\tilde{r}_{j-1 / 2}=\frac{\log \zeta_{j}-\log \zeta_{j-1}}{2 h}=\frac{1}{2 h} \log \left(\frac{\zeta_{j}}{\zeta_{j-1}}\right)
$$

or

$$
\tilde{r}_{j-1 / 2}=\frac{\left(\frac{\zeta_{j}^{1 / 2}-\zeta_{j-1}^{1 / 2}}{h}\right)}{\left(\frac{\zeta_{j}^{1 / 2}+\zeta_{j-1}^{1 / 2}}{2}\right)}=\frac{2}{h}\left(\frac{\zeta_{j}^{1 / 2}-\zeta_{j-1}^{1 / 2}}{\zeta_{j}^{1 / 2}+\zeta_{j-1}^{1 / 2}}\right)
$$

which formally lead to second-order numerical schemes.

Regarding the implicit relation between $D$ and $U$ we solve a $2 \times 2$ system to get that

$$
\left(\begin{array}{l}
D_{j}^{n+1} \\
U_{j-1}^{n+1}
\end{array}\right)=\left[\begin{array}{cc}
\tilde{\tau}_{j-1 / 2} & -\tilde{\sigma}_{j-1 / 2} \\
\tilde{\sigma}_{j-1 / 2} & \tilde{\tau}_{j-1 / 2}
\end{array}\right]\left(\begin{array}{c}
D_{j-1}^{n} \\
U_{j}^{n}
\end{array}\right)
$$


where

$$
\begin{gathered}
\tilde{\tau}_{j-1 / 2}=\frac{1-\left(h^{2} / 4\right) \tilde{r}_{j-1 / 2}^{2}}{1+\left(h^{2} / 4\right) \tilde{r}_{j-1 / 2}^{2}}, \\
\tilde{\sigma}_{j-1 / 2}=\frac{h \tilde{r}_{j-1 / 2}}{1+\left(h^{2} / 4\right) \tilde{r}_{j-1 / 2}^{2}},
\end{gathered}
$$

are the discrete transmission and reflection coefficients, respectively. A 1D Anderson localization study on a lattice uses a similar matrix structure [32]. Therefore, the matrix above defines the discrete reflection-transmission matrix at the staggered mesh point $x_{j-1 / 2}$.

Since these reflection-transmission matrices are orthogonal we obtain the following discrete analog for the energy conservation law

$$
\sum_{j \in \mathbb{Z}}\left(\left(D_{j}^{n+1}\right)^{2}+\left(U_{j}^{n+1}\right)^{2}\right)=\sum_{j \in \mathbb{Z}}\left(\left(D_{j}^{n}\right)^{2}+\left(U_{j}^{n}\right)^{2}\right) .
$$

For the sake of simplicity we are considering the entire line. Below we will add perfectly radiating boundary conditions. Therefore, the schemes presented are conservative and consequently stable in the discrete $L^{2}$-norm. Thus taking into account that it is second-order accurate, convergence follows directly from stability and consistency of the scheme. However, since we deal with rapidly varying acoustic media and high frequency wave fields (cf. equation (3.1)) accuracy in fact depends on how well the high frequency modes are represented by the computational grid. Furthermore, as we are interested in wave propagation in a random medium it is important to note that an appropriate mesh size selection (that depends on the introduced small parameter $\varepsilon$ ) allows us to reproduce the relevant statistical features of its corresponding continuous model.

We call attention to the analogy between the relations above and the equations for a Goupillaud medium (see the appendix A). Specifically, by using the approximation (4.3), the reflection-transmission matrices of the scheme and a Goupillaud layered medium coincide. In this case the numerical scheme can be interpreted as approximating the acoustic medium by a Goupillaud medium. The Goupillaud medium is frequently used in Geophysics.

As a final comment we point out that perfectly matching boundary conditions are easily implemented through the Riemann invariant formulation. We allow the computational domain to be homogeneous near its extreme points. Then the reflectivity $\tilde{r}$ is identically zero over these lattice points and the equations in system (4.1) decouple over these points. As a consequence of the decoupling, for example at the extreme right of the domain, $D$ propagates out of discrete model without generating any spurious reflection. This is clearly seen by (4.1b), with $j$ in the (extreme) homogeneous segment, implying that

$$
U_{j-1}^{n+1}=U_{j}^{n}
$$

and observing that $U_{j}^{n} \equiv 0$ there (recall that the impinging pulse is coming from the left). Hence, by the initial configuration, the left propagating Riemann invariant $U$ will always be zero at the extreme right of the domain. This is a guarantee that no spurious reflection will be generated at the boundary. We have tested the radiation of waves through the boundary in the homogeneous medium case and it worked very well. 
4.2. Operator splitting scheme. System (2.8) can be re-written in a more compact operator notation

$$
\partial_{t} \mathbf{a}=\mathrm{H}\left(x, \partial_{x}\right) \mathbf{a}
$$

where

$$
\mathbf{a}=\left(\begin{array}{l}
D \\
U
\end{array}\right) \quad \text { and } \quad \mathrm{H}\left(x, \partial_{x}\right)=\left[\begin{array}{cc}
-\partial_{x} & -r(x) \\
r(x) & \partial_{x}
\end{array}\right] .
$$

The operator $\mathrm{H}$ can be decomposed as the sum of a constant-coefficient diagonal hyperbolic operator $\mathrm{H}_{0}$ and a multiplicative operator $\mathrm{M}$ defined as

$$
\mathrm{H}_{0}\left(\partial_{x}\right)=\left[\begin{array}{cc}
-\partial_{x} & 0 \\
0 & \partial_{x}
\end{array}\right] \text { and } \mathrm{M}(x)=\left[\begin{array}{cc}
0 & -r(x) \\
r(x) & 0
\end{array}\right],
$$

with associated evolution equations

$$
\partial_{t} \mathbf{a}=\mathrm{H}_{\mathbf{0}} \mathbf{a} \text { and } \partial_{t} \mathbf{a}=\mathrm{Ma},
$$

respectively. We assume that $r(x)$ is continuous and bounded. The evolution (solution) operators $\mathcal{H}_{0}$ and $\mathcal{M}$ associated to equations (4.6) are unitary as in the lattice problem given in [32]. Their action on a vector valued function is readily available and we have

$$
\begin{gathered}
\mathcal{H}_{0}(t)\left(\begin{array}{l}
D(x) \\
U(x)
\end{array}\right)=\left(\begin{array}{c}
D(x-t) \\
U(x+t)
\end{array}\right), \\
\mathcal{M}(t)\left(\begin{array}{l}
D(x) \\
U(x)
\end{array}\right)=\left[\begin{array}{cc}
\cos (r(x) t) & -\sin (r(x) t) \\
\sin (r(x) t) & \cos (r(x) t)
\end{array}\right]\left(\begin{array}{l}
D(x) \\
U(x)
\end{array}\right) .
\end{gathered}
$$

Introducing a temporal grid with time step $\Delta t$ and using the Strang splitting scheme [34], we obtain the semi-discrete conservative scheme

$$
\mathbf{a}^{n+1}=\mathcal{H}_{0}\left(\frac{\Delta t}{2}\right) \mathcal{M}(\Delta t) \mathcal{H}_{0}\left(\frac{\Delta t}{2}\right) \mathbf{a}^{n} .
$$

Note that no approximation have been performed in space. For initial data and reflectivity coefficient sufficiently regular, it is second order accurate in time, thus convergence in the $L^{2}$-norm follows. Moreover, if $r(x)$ is only continuous and bounded the scheme is consistent [31] and consequently convergent.

For the discrete model applications we introduce spatial mesh points $\left\{x_{j}=\right.$ $j \Delta x \mid j \in \mathbb{Z}\}$ and adopt approximate values $\tilde{r}(x)$ for the reflectivity coefficient. Therefore we obtain the discrete evolution operator $\tilde{\mathcal{M}}$. After replacing it in (4.7) we get the splitting scheme

$$
\mathbf{a}^{n+1}=\mathcal{H}_{0}\left(\frac{\Delta t}{2}\right) \tilde{\mathcal{M}}(\Delta t) \mathcal{H}_{0}\left(\frac{\Delta t}{2}\right) \mathbf{a}^{n} .
$$

At this stage the medium has been discretized but not the solution modes $D$ and $U$. This discrete model is also conservative, but inherits truncation errors from the space discretization. For instance, using a central difference approximation for the reflectivity coefficient it becomes second order in space and time. 
By using a time step $\Delta t=\Delta x$ we avoid the introduction of interpolation errors since the characteristic lines go through the mesh points. Going through a complete discretization also in $D$ and $U$, and rearranging terms, the semi-discrete expression above takes the form

$$
\left(\begin{array}{c}
D_{j}^{n+1} \\
U_{j-1}^{n+1}
\end{array}\right)=\left[\begin{array}{cc}
\cos \left(\tilde{r}_{j-1 / 2} \Delta t\right) & -\sin \left(\tilde{r}_{j-1 / 2} \Delta t\right) \\
\sin \left(\tilde{r}_{j-1 / 2} \Delta t\right) & \cos \left(\tilde{r}_{j-1 / 2} \Delta t\right)
\end{array}\right]\left(\begin{array}{c}
D_{j-1}^{n} \\
U_{j}^{n}
\end{array}\right) .
$$

We again have a corresponding discrete reflection-transmission matrix as in [32]. Moreover, by approximating $\cos \left(\tilde{r}_{j-1 / 2} \Delta t\right)$ and $\sin \left(\tilde{r}_{j-1 / 2} \Delta t\right)$ by their second order Padé approximants, we obtain (4.4). Furthermore, the convergence analysis that we carry out in appendix B is also valid for this discrete model.

\section{Time-reversal refocusing experiments}

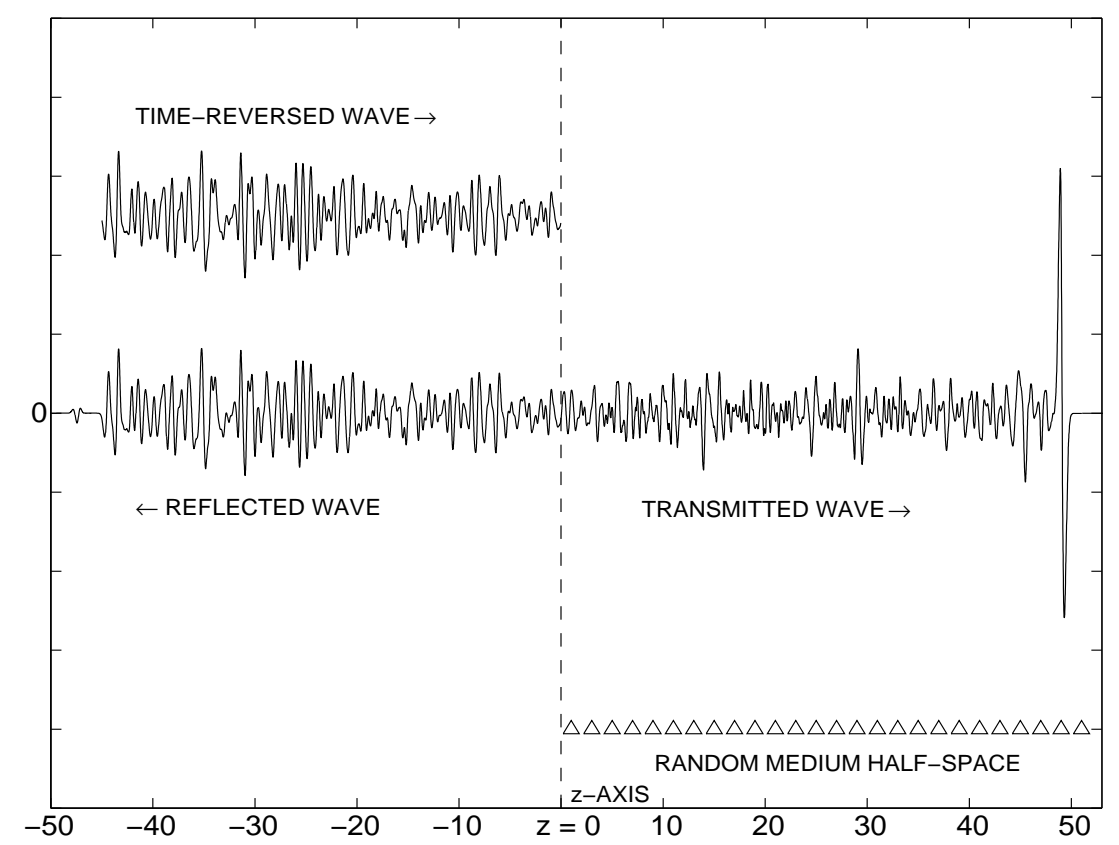

FIG. 5.1. A snapshot of a forward computational experiment together with the schematic indication of the time- reversal procedure. The leading wavefront is a right propagating pulse followed by fluctuations. By schematic we mean that the recording time indicated here is much shorter than in the experiments that follow, only to facilitate the visualization of the signal's fluctuations. The signal in the negative part of the domain is out of the computational domain and has been recorded in time as indicated by the (vertically) shifted signal. This signal is time-reversed and sent back into the random medium as a time dependent boundary condition along the (left) incoming characteristic.

In this section we present numerical illustrations of the refocusing of time-reversed reflections. For $h \ll 1$, small enough, all discrete models give similar results. We present the results for model (4.1) with the discrete reflectivity given by (4.2).

The numerical experiments are conducted as follows:

1. we let a pulse-shaped wave impinge upon the random medium from the left.

2. we record the reflected wave at the left extreme as a time signal, and re-enter it into the medium after time-reversion, by considering it as a time-dependent 
boundary condition along the incoming characteristic (cf. Fig. 5.1). Along the homogeneous region the incoming and outgoing signals $(D$ and $U)$ are decoupled.

An equivalent procedure, frequently used, consists of capturing a spatial segment of the reflected signal traveling to the left in the homogeneous half-space, at a particular instant of time; 'freezing' this piece and sending it back into the heterogeneous medium as a secondary right propagating wave.

Since we shall compute the approximate solution for a finite time interval, from the hyperbolicity of the equations follows that only a slab of the heterogeneous halfspace should be considered. Then the domain of interest is completed by adding little pieces of homogeneous medium to both sides of this slab, in order to correctly manage the radiation boundary conditions and also properly setting the initial incoming pulse/signal.

The heterogeneous (slab) medium properties are modeled by considering a constant compressibility $\kappa_{0}$ and setting random density values at points $\tilde{z}_{j}=j \varepsilon$ of the physical domain. This is done using a random number generator, so that $\rho\left(\tilde{z}_{j}\right)=\rho_{0}\left(1+Y_{j}\right)$ where $Y_{j}$ are independent random variables uniformly distributed over $(-C, C)$ with $0<C<1$. This ensures that the fluctuations correlation length is $O(\varepsilon)$. Connecting these values we define the medium density as a piecewise linear function over the whole slab. For simplicity we set $\varrho_{0}=\kappa_{0}=1$ in all numerical experiments but section 5.2.

The computational mesh is constructed as follows: first the change of variables (2.3) is performed by mapping analytically the points $\tilde{z}_{j}$, of the physical domain, into their travel time images $\tilde{x}_{j}$ in the computational domain. Then by a linear interpolation we obtain approximately the points $z_{k}$ that map to the uniform mesh defined by $x_{k}=k h$ and set the corresponding densities to $\rho_{k}=\rho\left(z_{k}\right)$.

Concerning the mesh size $h$, a choice of 5-10 mesh points per correlation length (or layer) gives excellent results. This was also observed in the numerical experiments presented in [4]. In $[23,33]$ a spatial discretization with mesh size equal $\varepsilon$ also produced very accurate results. This is in agreement with the analysis presented in appendix B.

We again remark that when the signal is recorded in space we need to use a mesh sufficiently large to accommodate for the entire reflected signal at the left side of the heterogeneous slab (as indicated in Fig. 5.1). Therefore, the size of the computational domain increases proportionally to the recorded time $t_{0}$. However, the procedure implemented in our experiments uses a computational domain roughly of the size of the slab, independently of the length of the recording interval. This is due to the exact radiation condition defined through the (corresponding) outgoing characteristics.

In all the experiments that follow we take $\varepsilon=0.1$, and a mesh size $h=0.01$. The heterogeneous slab has length $L$ and the initial pulse is given by the Gaussian derivative

$$
f(z)=-7(z-2) \exp \left(-\frac{(z-2)^{2}}{0.1}\right) .
$$

Note that its effective support is $O(1)$, and consequently the medium correlation length to pulse width ratio is $O(\varepsilon)$. As a cutoff function for the time-reversal mirror we take the indicator function of the time interval $\left[0, t_{0}\right]$. The recording time for all numerical experiments is large, corresponding to the propagation from 50 up to 400 initial pulse widths. We point out that this setting is in complete agreement with the scaling discussed in section 3 . 
5.1. Complete refocusing and localization (Figures 5.2-5.3). In this first experiment, our goal is to illustrate the complete refocusing of the time-reversed pulse. As we pointed out before, this is closely related to Anderson localization.

We use a heterogeneous slab with length $L=405$, having small homogeneous segments at its extremes. The forward experiment, with the initial pulse generating (backscattered) reflections, is followed by the time-reversed experiment using the recorded reflected signal as initial data.

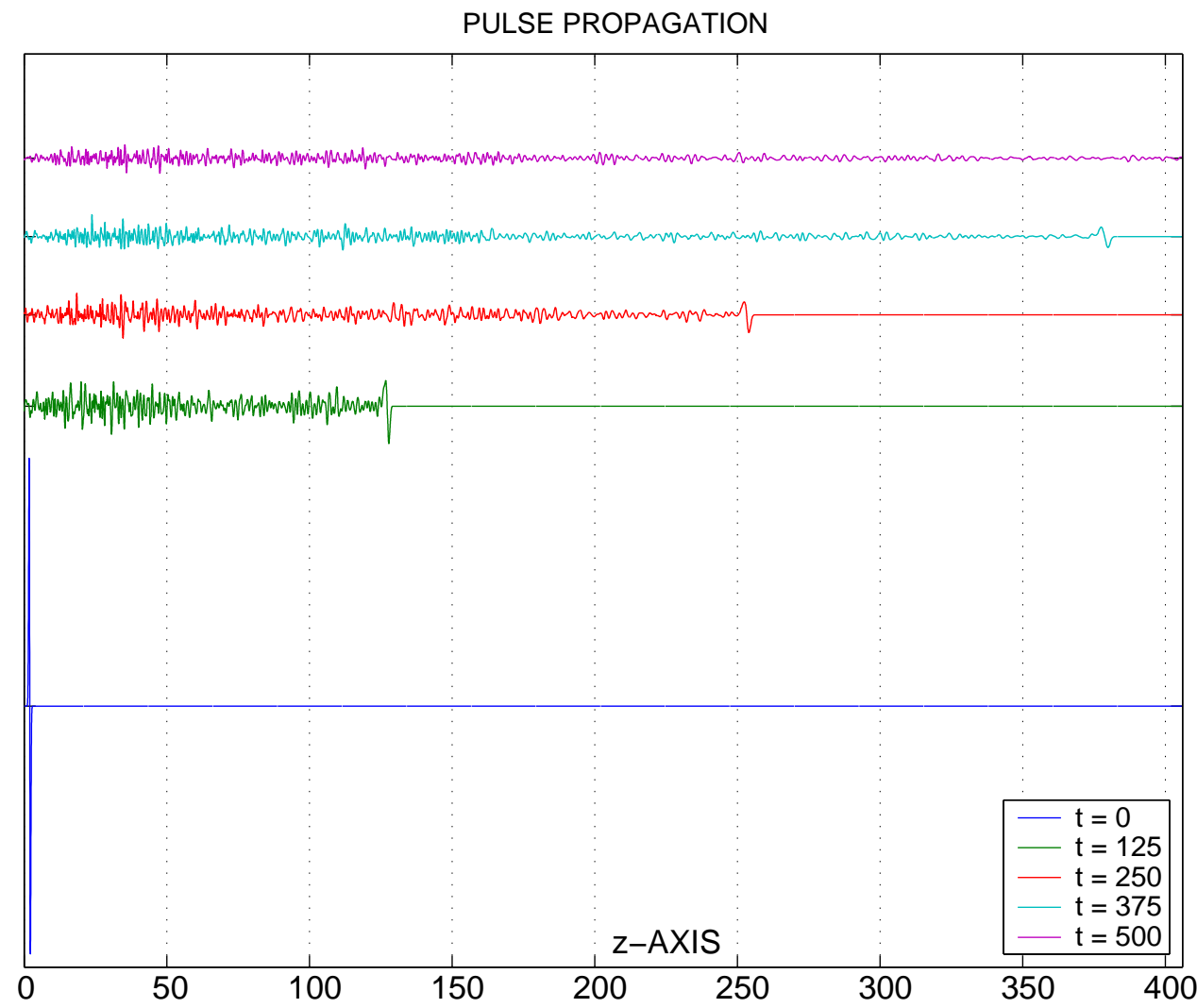

FIG. 5.2. Forward experiment: the initial pulse profile is located at the left of the bottom trace and seen as a vertical line near the origin. This is due to the long horizontal scale of the graph (a total of 400 pulse widths). A detail of the initial pulse (of approximately unit width) is provided in Fig. 5.3. The right propagating pulse evolves as displayed at different times $t$. Traces from bottom to top are: $t=0,125,250,375$ and 500. The attenuation and broadening of the pulse, along the wavefront, is clearly seen to the right of the snapshots for $t \geq 125$.

Fig. 5.2 shows the forward experiment with the O'Doherty-Anstey attenuation taking place along the wavefront $[3,30]$ as a manifestation of Anderson localization. Time evolves from the bottom of the figure towards the top. At the bottom left (near zero) we have the initial (Gaussian derivative) pulse. As time evolves we see the diffusive-like behavior along the leading pulse. Very little energy is being transmitted at the right end of the computational domain. Moreover, the higher-frequency modes stay closer to the extreme left of the slab and their amplitudes clearly decay with time. This indicates that they scatter back through the interface. At time $t=500$ (the top trace) the small leading wavefront has propagated out of the computational 
domain without generating any spurious reflections as explained earlier. The reflected signal is recorded at the left end as it leaves the computational domain.

In order to see better how good the recompression is we compare two time-reversed experiments: one for which we recorded the reflected signal for $t_{0}=410$ and another which we recorded for a time interval twice as long $\left(t_{0}=820\right)$. The result is shown in Fig. 5.3 where we compare the refocused pulses with the initial profile. It is clear that we have been able to recompress (effectively) the initial data's energy. As mentioned above, this is predicted by the theory (c.f. expression (3.8)) and is a manifestation of localization: if the random medium is long enough we have zero transmission (the medium is opaque).

In fact, the recompressed energy measured as $\left\|U_{0}^{\varepsilon, T R}\left(t_{0}+\cdot\right)\right\|_{2} /\|f\|_{2}$ increases from $89.9 \%$ to $94.4 \%$ when $t_{0}=410$ and $t_{0}=820$, respectively. Moreover, the unrecovered portion of the initial pulse quantified as $\Delta=\left\|U_{0}^{\varepsilon, \mathrm{TR}}\left(t_{0}+\cdot\right)-f(-\cdot)\right\|_{\infty} /\|f\|_{\infty}$ falls from $\Delta_{1}=12.6 \%$ when $t_{0}=410$ to $\Delta_{2}=8.5 \%$ for $t_{0}=820$, and their ratio $\Delta_{1} / \Delta_{2}=1.47$ appears to be very close to the predicted value $\sqrt{2}$ (c.f. (3.8)). The value $\sqrt{2}$ comes from the fact that we doubled the recording time in the second experiment.

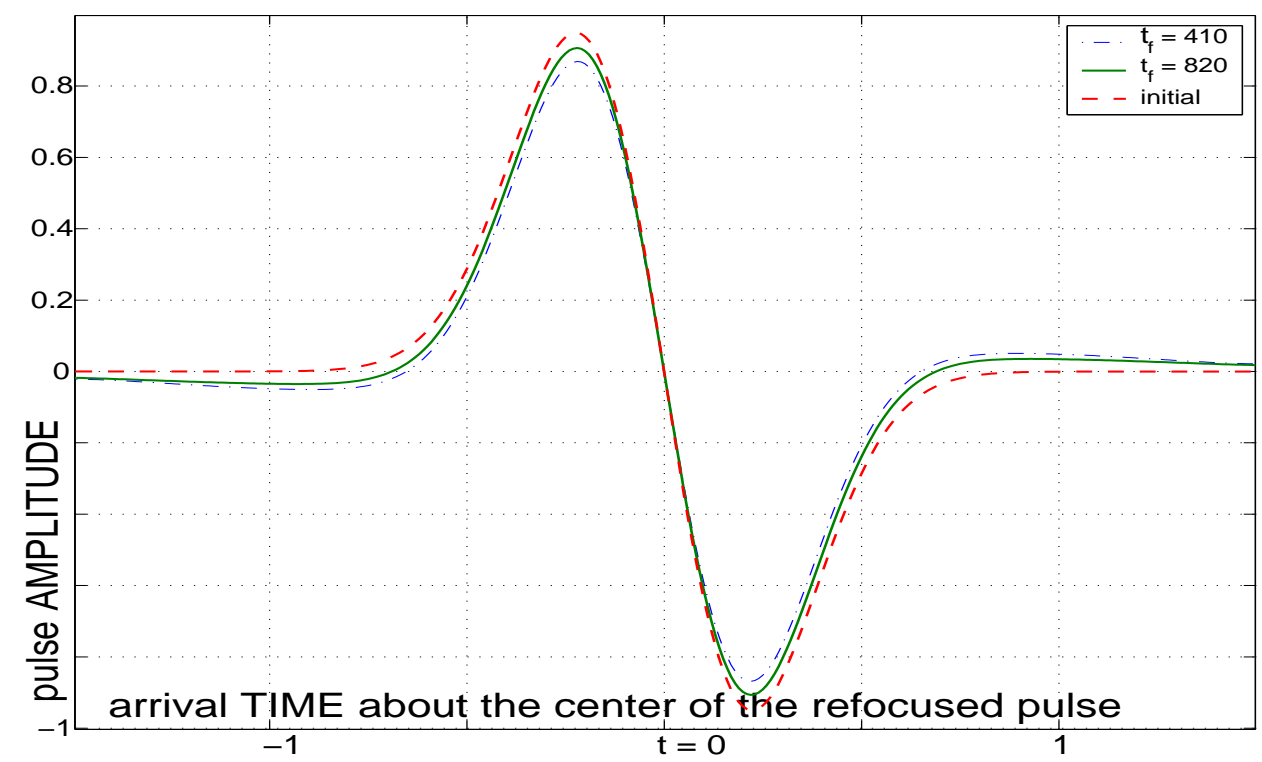

FIG. 5.3. Initial profile (dashed line) and refocused pulses for $t_{0}=410$ (dash-dotted line) and $t_{0}=820$ (solid line).

5.2. Background medium effect (Figure 5.4-5.6). Now we experiment with a scenario that has not been explored before. In particular we will give evidence on the robustness of the refocusing phenomenon by gradually going against the separation of scales hypothesis regarding the wave and the medium, which is very important for developing the theory. In this example the background medium is not homogeneous: we define the background medium density by $\varrho_{0}(z)=1+0.5 \sin (2 \pi m z / L)$ on a slab $[4,84]$ of length $L=80$ and set $m=4,20$ and 80 . The physical domain extends from $z_{i}=0$ to $z_{f}=86$ and the recording time $t_{0}=90$. In Fig. 5.4 we present the numerical results for a wide range of background scales. In particular for $m=80$ the deterministic background scale is of the same order as the pulse. We note that as 
the medium varies on faster scale the refocused pulse has a larger amplitude. In any case the robustness of the incoherence recompression phenomenon (i.e. refocusing in reflection) is remarkable.

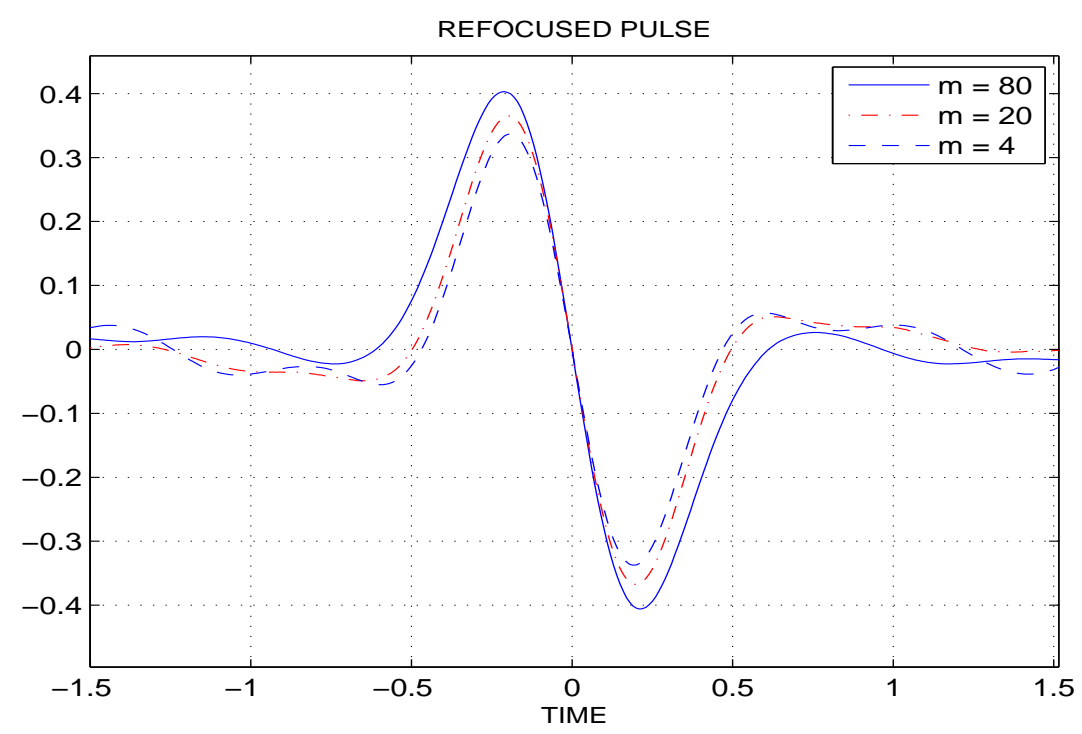

FIG. 5.4. Refocusing in the case of a varying background. We show the refocused pulses for $m=4$ (dashed line), $m=20$ (dash-dotted line) and $m=80$ (solid line).

The statistical stabilization of the refocused pulse has been theoretically established only for a slowly varying background. Thus, our numerical simulations when $m=4$ and 20 are consistent with this result even though we do not present this result here. Moreover, from the results shown in Fig. 5.5, corresponding to time-reversal experiments in the case where $m=80$, it is apparent that for this faster background the refocused pulse is still statistically stable.

Furthermore, in Fig. 5.6 we show the averaged refocused pulses (over 10 realizations of the random medium) when $m=4,20$ and 80 . It is remarkable that for $m=4$ and 20, the refocused pulses are almost identical while for $m=80$ it has a larger amplitude. So, the fast periodic variations of the background enhances the quality of the recompressed pulse amplitude.

We believe that this enhancement is a manifestation of the celebrated Bragg resonance for waves propagating over periodic structures (see for instance [8]). Indeed, the periodic background is able to reflect back a portion of the energy carried by the Fourier modes whose wavelengths are close to the resonant wavelength (equal to twice the background period). Moreover, due to the separation of scales within the medium (i.e. the periodic profile and the random fluctuations) this effect is not annihilated by the random fluctuations. Therefore, there is more energy available for recompression and it leads to an improved refocused pulse. Nevertheless, a more detailed analysis regarding the discussed effect is needed.

5.3. Wave train refocusing (Figure 5.7). In this experiment we consider the refocusing of a pulse shaped wave train in the form of a bit stream, as presented at the top trace given in Fig. 5.7. The objective of the example with the bit stream is to 


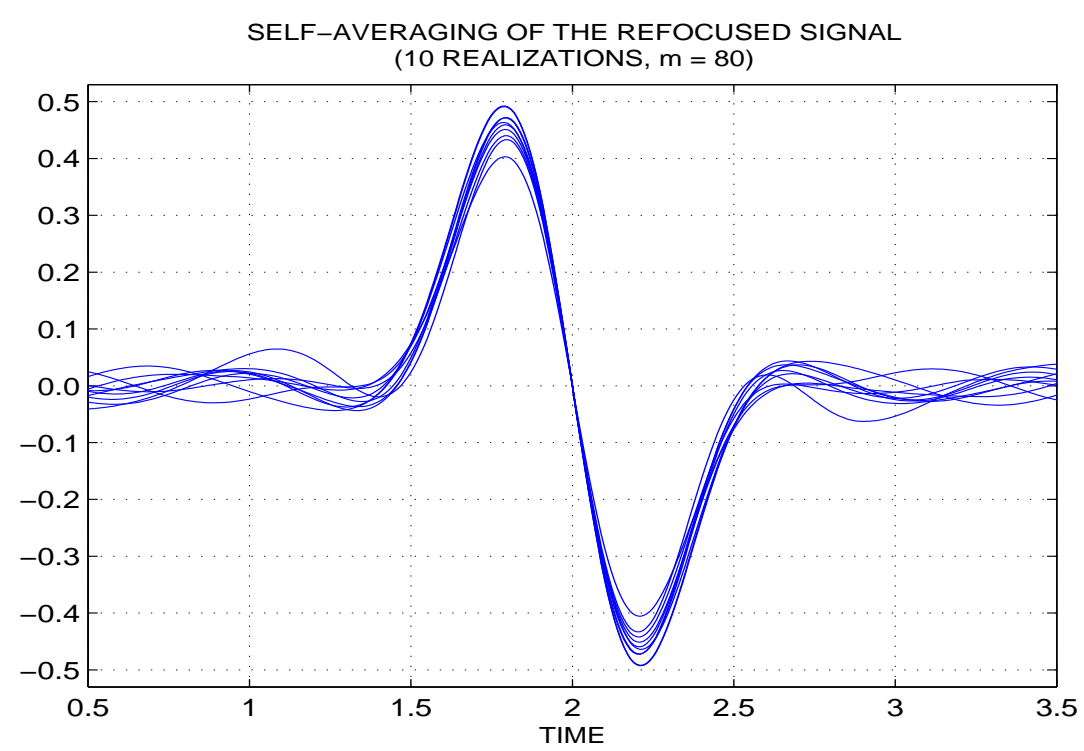

FIG. 5.5. Statistical stabilization of the refocused pulse corresponding to the case of a rapidly varying background $(m=80)$. The results of 10 realizations are shown.

show that, after being scrambled by random reflection, it can be unscrambled through time-reversal. As an example for scrambling transmitted signals one will find the timereversal in transmission technique given by Heinemann and Smith [26]. They consider $2 \mathrm{D}$ experimental results of a scheme, for encrypting acoustic communication in a reverberant environment. To the best of our knowledge this scrambling-unscrambling has never been done for 1D time-reversal in reflection. At this stage, our goal is to show how time-reversal unscrambling works in a nice and clean fashion. We hope that this might lead to interesting applications in the future.

The top trace in Fig. 5.7 is the wave train $f(t)$ corresponding to the bit stream $\beta=\beta_{1} \beta_{1} \ldots \beta_{64}$. Each (initial) unit bit is described by the derivative of the Gaussian $f_{0}$ given by (5.1) and the support of a zero bit is the same as the effective support of the unit bit. This initial pulse is given as $f(t)=\sum_{i=1}^{64} \beta_{i} f_{0}(t+2(i-1))$.

The middle trace in Fig. 5.7 represents the scrambled reflected signal generated by the interaction of the bit stream with a disordered acoustic medium. Recall that the reflected trace is recorded at the left end of the acoustic channel, namely along the left going characteristic.

The initial pulse shape (of each bit) and the bit stream order were now randomly scrambled through the forward experiment. We have used a heterogeneous slab of length $L=200$ within a physical domain from $z_{i}=0$ to $z_{f}=202$ and we set $t_{0}=485$. We then perform the time-reversal procedure for the incoherent time-signal given at the middle trace of Fig. 5.7. Recall that we do not need to allocate the reflected and refocused signals on the computational mesh. This long, incoherent, time-reversed signal is sent back into the random slab as a time-dependent boundary condition at the left end of our computational domain. This boundary condition is imposed along the incoming characteristic, from the left. Again the robustness of the refocusing phenomenon is clearly seen at the bottom trace of Fig. 5.7. The recompression 


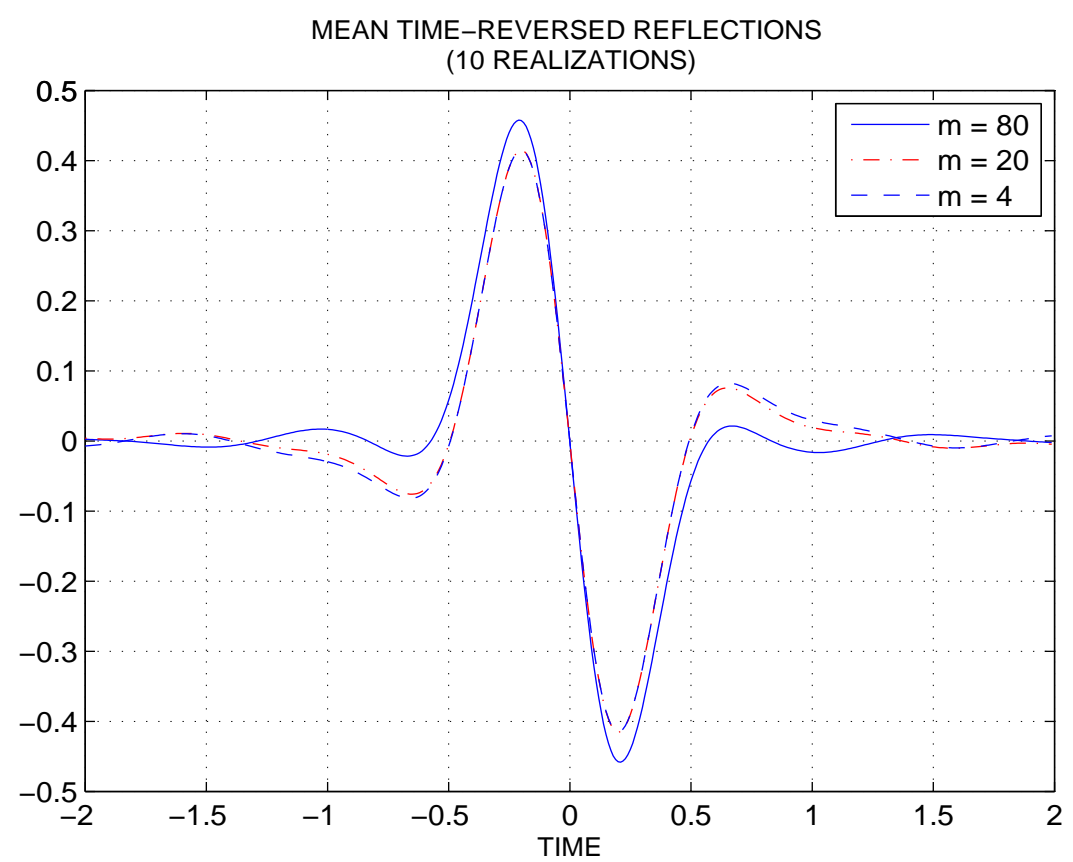

FIG. 5.6. Mean refocused pulses (averaging over 10 realizations of the random medium) for the case of a varying background. We show the refocused pulses for $m=4$ (dashed line), $m=20$ (dash-dotted line) and $m=80$ (solid line). Note that the first two signals are almost identical.

process is very efficient along the entire bit stream. In particular the zeros of the bit stream are recovered in a very "clean" fashion and we have a uniform amplitude distribution for the recompressed bits.

This looks paradoxical at first glance, as the reflected signal corresponding to the $i$-th bit is recorded from $t_{0, i}=2(i-1)$ to $t_{0}$ so there is less information available for recompressing the last non-zero bit than for the first one. However, since $t_{0, i} / t_{0}=O(\varepsilon)$ this quantity of information is negligible as $\varepsilon \rightarrow 0$. Moreover, if $f_{0}^{\mathrm{TR}}$ represents the limiting refocused pulse corresponding to $f_{0}$ then from (3.4) one gets that $f^{\mathrm{TR}}(t)=$ $\sum_{i=1}^{64} \beta_{i} f_{0}^{\mathrm{TR}}(t+2(i-1))$. This is a wave train representing the bit stream $\beta$ where each unit bit is codified through $f_{0}^{\mathrm{TR}}$.

Appendix A. The Goupillaud layered medium. Goupillaud layered media are frequently used as geophysical models $[3,30]$. Due to the analogy mentioned in section 4, for the lagrangian schemes and the transmission-reflection relations for a layered medium, in this section we describe a Goupillaud layered medium. Here $\varrho(z)$ and $\kappa(z)$ are piecewise constant with jump discontinuities at interfaces, in such a way that the travel time across every layer is the same.

Therefore the interfaces are uniformly spaced at intervals of $\Delta x$ in the travel time coordinate $x$ and we use the notation

$$
x_{j}=j \Delta x, \quad f_{j}(\ldots)=f\left(x_{j}, \ldots\right), \quad j \in \frac{1}{2} \mathbb{Z},
$$

for any function dependent on $x$. 
SIGNAL PROFILES

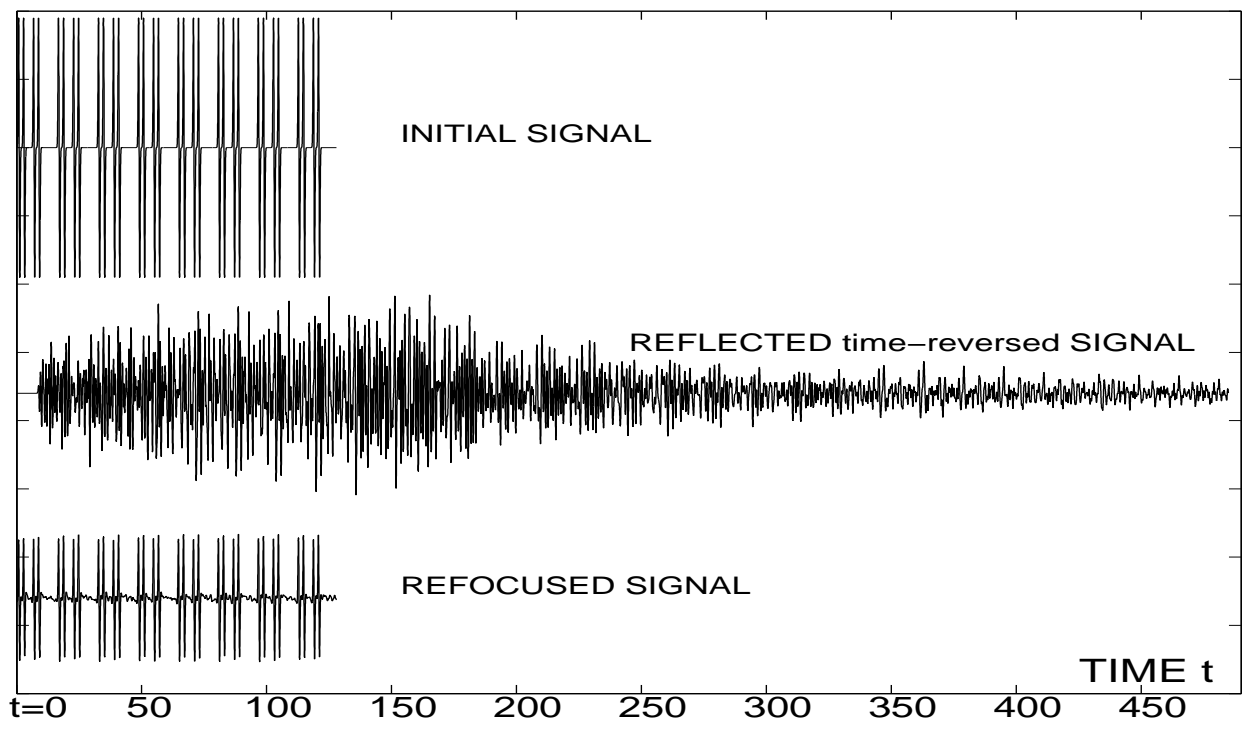

FIG. 5.7. Wave train refocusing. The horizontal axis is time for a signal recorded at the left end of the computational domain. The initial profile is presented at the top trace representing the bit stream 1101100011011000110110001101100011011000110110001101100011011000. The incoherent reflected signal is presented at the middle trace and the (unscrambled) refocused wave train at the bottom trace. The original bit stream is recovered.

Now, without loss of generality we consider the interfaces positioned at points $x_{j-1 / 2}, j \in \mathbb{Z}$ and by assuming the continuity of the pressure and velocity wavefields, from equations (2.7) we get the following jump conditions for the right- and left-side limits of $D(x, t)$ and $U(x, t)$, respectively, at the interface $x=x_{j-1 / 2}$

$$
\left[\begin{array}{cc}
\zeta_{j-1}^{-1 / 2} & -\zeta_{j-1}^{-1 / 2} \\
\zeta_{j-1}^{1 / 2} & \zeta_{j-1}^{1 / 2}
\end{array}\right]\left(\begin{array}{c}
D_{j-1 / 2-0}(t) \\
U_{j-1 / 2-0}(t)
\end{array}\right)=\left[\begin{array}{cc}
\zeta_{j}^{-1 / 2} & -\zeta_{j}^{-1 / 2} \\
\zeta_{j}^{1 / 2} & \zeta_{j}^{1 / 2}
\end{array}\right]\left(\begin{array}{c}
D_{j-1 / 2+0}(t) \\
U_{j-1 / 2+0}(t)
\end{array}\right) .
$$

Moreover, since in each layer the underlying medium is homogeneous the Riemann invariants are conserved along the segments of characteristic lines between the interfaces, and by continuity

$$
\begin{aligned}
& D_{j-1 / 2-0}(t+\Delta t / 2)=D_{j-1}(t) \\
& D_{j-1 / 2+0}(t+\Delta t / 2)=D_{j}(t+\Delta t) \\
& U_{j-1 / 2-0}(t+\Delta t / 2)=U_{j-1}(t+\Delta t) \\
& U_{j-1 / 2+0}(t+\Delta t / 2)=U_{j}(t)
\end{aligned}
$$

where we have set $\Delta t=\Delta x$. Finally, by evaluating in (A.1) for time $t+\Delta t / 2$ and using the relations above we obtain the system

$$
\left(\begin{array}{c}
D_{j}(t+\Delta t) \\
U_{j-1}(t+\Delta t)
\end{array}\right)=\left[\begin{array}{cc}
\tau_{j-1 / 2} & -\sigma_{j-1 / 2} \\
\sigma_{j-1 / 2} & \tau_{j-1 / 2}
\end{array}\right]\left(\begin{array}{c}
D_{j-1}(t) \\
U_{j}(t)
\end{array}\right)
$$

where the matrix standing in the right hand side is known as the reflectiontransmission matrix with the transmission and reflection coefficients defined, respec- 
tively, by

$$
\begin{aligned}
\tau_{j-1 / 2} & =2 \sqrt{\zeta_{j} \zeta_{j-1}} /\left(\zeta_{j}+\zeta_{j-1}\right) \\
\sigma_{j-1 / 2} & =\left(\zeta_{j}-\zeta_{j-1}\right) /\left(\zeta_{j}+\zeta_{j-1}\right) .
\end{aligned}
$$

Since $\tau_{j-1 / 2}^{2}+\sigma_{j-1 / 2}^{2}=1$, the reflection-transmission matrices are orthogonal and we get the energy conservation law for the Goupillaud medium in the form

$$
\sum_{j \in \mathbb{Z}}\left(D_{j}^{2}(t)+U_{j}^{2}(t)\right)=\sum_{j \in \mathbb{Z}}\left(D_{j}^{2}(t+\Delta t)+U_{j}^{2}(t+\Delta t)\right) .
$$

These equations allow us to characterize the solution at any point in space and time. More precisely, we get the solution at the midpoint of the layers using steps in time equal to the travel time between interfaces. Note that all the analysis above can be made by setting $\Delta x$ equal to an integer fraction of the travel time across the layers.

Appendix B. Convergence analysis of the discrete model. In this section we carry out an analysis of the discrete models introduced in this work to establish that some important features of the continuous model given by the acoustic wave equations are very well reproduced. In fact, we show that as $\varepsilon \rightarrow 0$, the limiting discrete (keeping $\delta=h / \varepsilon$ constant) and continuous signals reflected at the interface of the random medium give rise to mean zero stationary random processes with similar covariance functions. Furthermore, as $\delta \rightarrow 0$ this discrete covariance function approaches the continuous one.

In this analysis, we assume that the reflectivity coefficient can be expressed as

$$
\tilde{r}_{j-1 / 2}=\frac{\varepsilon^{1 / 2}}{h}\left(\mu\left(\frac{j h}{\varepsilon}\right)-\mu\left(\frac{(j-1) h}{\varepsilon}\right)\right)
$$

where $\mu(\cdot)$ is a bounded, mean-zero stationary, and exponentially mixing random process with correlation length of $O(1)$. Consequently, the discrete reflection coefficient can be written as

$$
\tilde{\sigma}_{j-1 / 2}=\varepsilon^{1 / 2}\left(\mu\left(\frac{j h}{\varepsilon}\right)-\mu\left(\frac{(j-1) h}{\varepsilon}\right)\right)+O(\varepsilon) .
$$

Our goal is to characterize the scattered signal generated by an incident pulse of the form $D(0, t)=\varepsilon^{-1 / 2} f\left(\frac{t}{\varepsilon}\right)$ where $f(\cdot) \in L^{2}(\mathbb{R})$. The analysis relies on an appropriate Fourier representation of the scattered signal, together with the asymptotic characterization of some statistics of the reflection coefficient in the frequency domain.

We start by introducing an $\varepsilon$-scaled version of the semi-discrete Fourier transform corresponding to the function $g \in L^{2}(h \mathbb{Z})$ as follows

$$
\hat{g}_{h}^{\varepsilon}(\omega)=\frac{h}{\sqrt{2 \pi}} \sum_{m=-\infty}^{\infty} \mathrm{e}^{\mathrm{i} \frac{m h \omega}{\varepsilon}} g(m h), \quad \omega \in\left[-\frac{\varepsilon \pi}{h}, \frac{\varepsilon \pi}{h}\right] .
$$

Consequently, the corresponding inversion formula is given by

$$
g(m h)=\frac{1}{\sqrt{2 \pi} \varepsilon} \int_{-\frac{\varepsilon \pi}{h}}^{\frac{\varepsilon \pi}{h}} \mathrm{e}^{-\mathrm{i} \frac{m h \omega}{\varepsilon}} \hat{g}_{h}^{\varepsilon}(\omega) \mathrm{d} \omega, \quad h \in \mathbb{Z} .
$$

Note that when $\varepsilon=1$ this coincides with the usual semi-discrete Fourier transform in $L^{2}(h \mathbb{Z})$ represented as $\hat{g}_{h}(\cdot)$. 
By using the semi-discrete Fourier transform, and introducing the unknowns $a_{m}^{\varepsilon}(\omega)=\mathrm{e}^{-\mathrm{i} \frac{m h \omega}{\varepsilon}} \hat{D}_{m}^{\varepsilon}(\omega), b_{m}^{\varepsilon}(\omega)=\mathrm{e}^{\mathrm{i} \frac{m h \omega}{\varepsilon}} \hat{U}_{m}^{\varepsilon}(\omega)$ from (4.4) we get that

$$
\left(\begin{array}{l}
a_{j}^{\varepsilon}(\omega) \\
b_{j}^{\varepsilon}(\omega)
\end{array}\right)=P_{j-1}^{\varepsilon}(\omega)\left(\begin{array}{l}
a_{j-1}^{\varepsilon}(\omega) \\
b_{j-1}^{\varepsilon}(\omega)
\end{array}\right), \quad j \in \mathbb{Z}
$$

where

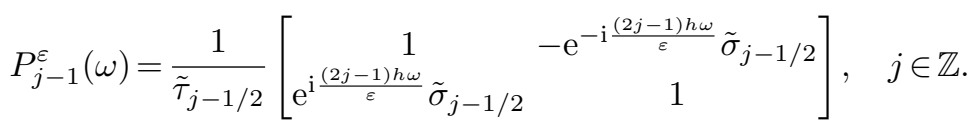

The reflection and transmission coefficients $R_{0}^{\varepsilon}(\omega), T_{0}^{\varepsilon}(\omega)$ (in the frequency domain) corresponding to the stack of layers from $j=0$ to $J=\lfloor L / h\rfloor$ (associated with the random slab $[0, L])$ are defined through the equations

$$
\left(\begin{array}{c}
T_{0}^{\varepsilon} \\
0
\end{array}\right)=Y_{0}^{\varepsilon}\left(\begin{array}{c}
1 \\
R_{0}^{\varepsilon}
\end{array}\right)
$$

where

$$
Y_{0}^{\varepsilon}=P_{J-1}^{\varepsilon} P_{J-2}^{\varepsilon} \cdots P_{0}^{\varepsilon}
$$

Using a discrete version of the invariant embedding technique and taking into account the form of the matrices $P_{j}^{\varepsilon}$ we obtain a discrete analog of not the Riccatti equations for these coefficients, namely that

$$
\begin{aligned}
T_{j-1}^{\varepsilon} & =\frac{\tilde{\tau}_{j-1 / 2} T_{j}^{\varepsilon}}{1-\mathrm{e}^{-\mathrm{i} \frac{(2 j-1) h \omega}{\varepsilon}} \tilde{\sigma}_{j-1 / 2} R_{j}^{\varepsilon}} \\
R_{j-1}^{\varepsilon} & =\frac{R_{j}^{\varepsilon}-\mathrm{e}^{\mathrm{i} \frac{(2 j-1) h \omega}{\varepsilon}} \tilde{\sigma}_{j-1 / 2}}{1-\mathrm{e}^{-\mathrm{i} \frac{(2 j-1) h \omega}{\varepsilon}} \tilde{\sigma}_{j-1 / 2} R_{j}^{\varepsilon}}
\end{aligned}
$$

with the final conditions $T_{J}^{\varepsilon}=1, R_{J}^{\varepsilon}=0$. In this context, the conservation of energy is expressed as $\left|R_{j}^{\varepsilon}\right|^{2}+\left|T_{j}^{\varepsilon}\right|^{2}=1$.

The concepts introduced above allow for the following representation of the (discrete) scattered signal $U_{0}^{m}=U(x=0, t=m h)$ reflected at the interface $x=0$ of the slab $[0, L]:$

$$
U_{0}^{m}=\frac{1}{\sqrt{2 \pi \varepsilon}} \int_{-\frac{\varepsilon \pi}{h}}^{\frac{\varepsilon \pi}{h}} \mathrm{e}^{-\mathrm{i} \frac{m h \omega}{\varepsilon}} R_{0}^{\varepsilon}(\omega) \hat{f}_{\frac{h}{\varepsilon}}(\omega) \mathrm{d} \omega
$$

This formula is analogous to the integral representation presented in [11].

Next, we study the asymptotic behavior when $\delta=h / \varepsilon=$ constant and $\varepsilon \rightarrow 0$ of the interpolated reflected signal $u_{t_{0}}^{\delta, \varepsilon}(\cdot)$ in a $\varepsilon$-scaled time window centered at time $t_{0}$, where

$$
u_{t_{0}}^{\delta, \varepsilon}(s)=U_{0}^{m}
$$

with $m=\left(t_{0}+\varepsilon s\right) / h$. A simple asymptotic expansion for the reflection coefficient yields $\mathbb{E}\left\{R_{0}^{\varepsilon}(\omega)\right\}=O(\varepsilon)$ and consequently $\lim _{\varepsilon \rightarrow 0} \mathbb{E}\left\{u_{t_{0}}^{\delta, \varepsilon}(\cdot)\right\}=0$. For the covariance 
$C_{t_{0}}^{\delta, \varepsilon}\left(s_{1}, s_{2}\right)=\mathbb{E}\left\{u_{t_{0}}^{\delta, \varepsilon}\left(s_{1}\right) \overline{u_{t_{0}}^{\delta, \varepsilon}\left(s_{2}\right)}\right\}$, after some transformations, we get the following representation

$$
\begin{aligned}
C_{t_{0}}^{\delta, \varepsilon}\left(s_{1}, s_{2}\right)=\frac{1}{2 \pi} \int_{-\frac{\pi}{\delta}}^{\frac{\pi}{\delta}} \mathrm{d} \omega \mathrm{e}^{\mathrm{i}\left(s_{2}-s_{1}\right) \omega} \int_{-\frac{2}{\varepsilon}\left(\frac{\pi}{\delta}-|\omega|\right)}^{\frac{2}{\varepsilon}\left(\frac{\pi}{\delta}-|\omega|\right)} \mathrm{d} q \times \\
\quad \mathrm{e}^{-\mathrm{i}\left(t_{0}+\frac{s_{1}+s_{2}}{2} \varepsilon\right) q} \mathbb{E}\left\{R_{0}^{\varepsilon}\left(\omega+\varepsilon \frac{q}{2}\right) \overline{R_{0}^{\varepsilon}\left(\omega-\varepsilon \frac{q}{2}\right)}\right\} \hat{f}_{\delta}\left(\omega+\varepsilon \frac{q}{2}\right) \overline{\hat{f}_{\delta}\left(\omega-\varepsilon \frac{q}{2}\right)} .
\end{aligned}
$$

By applying a diffusion-approximation theorem (see section B.1 below) one gets that $\lim _{\varepsilon \rightarrow 0} \mathbb{E}\left\{R_{0}^{\varepsilon}\left(\omega+\varepsilon \frac{q}{2}\right) \overline{R_{0}^{\varepsilon}\left(\omega-\varepsilon \frac{q}{2}\right)}\right\}=w_{\delta}(\omega, q)$ to be defined in the sequel. Consequently, we have that

$$
C_{t_{0}}^{\delta}\left(s_{2}-s_{1}\right)=\lim _{\varepsilon \rightarrow 0} C_{t_{0}}^{\delta, \varepsilon}\left(s_{1}, s_{2}\right)=\int_{-\frac{\pi}{\delta}}^{\frac{\pi}{\delta}} \mathrm{e}^{\mathrm{i}\left(s_{2}-s_{1}\right) \omega} \Lambda_{\delta}\left(t_{0}, \omega\right)\left|\hat{f}_{\delta}(\omega)\right|^{2} \mathrm{~d} \omega
$$

where $\Lambda_{\delta}(t, \omega)$ is the (continuous) inverse Fourier transform of $w_{\delta}(\omega, q)$ with respect to $q$. We establish in this way that the limiting re-scaled scattered signal is a stationary centered random process with power spectral density given by $\Lambda_{\delta}\left(t_{0}, \omega\right)\left|\hat{f}_{\delta}(\omega)\right|^{2}$. Furthermore, the normalized spectral density is expressed as

$$
\Lambda_{\delta}(t, \omega)=\frac{\Gamma_{\mu}^{\delta}(\omega)}{\left(1+\Gamma_{\mu}^{\delta}(\omega) t\right)^{2}}
$$

where

$$
\Gamma_{\mu}^{\delta}(\omega)=\frac{1}{\delta}\left\{\sin ^{2}(\omega \delta) \sum_{k=0}^{\infty} R_{\mu}(k \delta) \cos (2 \omega k \delta)+\frac{R_{\mu}(0) \cos (2 \omega \delta)-R_{\mu}(\delta)}{4}\right\}
$$

with $R_{\mu}(s)=\mathbb{E}\{\mu(s) \mu(0)\}$.

We have to note that in the limit when $\delta \rightarrow 0$, we have that $\Gamma_{\mu}^{\delta}(\omega) \rightarrow \omega^{2} \gamma_{\mu}^{c}(\omega)$ pointwise, where $\gamma_{\mu}^{c}(\omega)=\int_{0}^{+\infty} R_{\mu}(s) \cos (2 \omega s) \mathrm{d} s$. It follows that, $\Lambda_{\delta}(t, \omega) \rightarrow \Lambda(t, \omega)$ where $\Lambda(t, \omega)=\frac{\omega^{2} \gamma_{\mu}^{c}(\omega)}{\left(1+\omega^{2} \gamma_{\mu}^{c}(\omega) t\right)^{2}}$, and since $\hat{f}_{\delta}(\omega) \rightarrow \hat{f}(\omega)$, one finally gets that

$$
\lim _{\delta \rightarrow 0} C_{t_{0}}^{\delta}\left(s_{2}-s_{1}\right)=\int_{-\infty}^{+\infty} \mathrm{e}^{\mathrm{i}\left(s_{2}-s_{1}\right) \omega} \Lambda\left(t_{0}, \omega ; \mu(\cdot)\right)|\hat{f}(\omega)|^{2} \mathrm{~d} \omega
$$

which is consistent with the results in $[3,10]$.

B.1. Computation of $\Lambda_{\delta}(t, \omega)$. The computation of the normalized spectral density $\Lambda_{\delta}$, of the limiting reflected signal, can be carried out along the same lines as in [9]. We will establish that in the limit $\varepsilon \rightarrow 0$, the statistics of the reflection coefficient $R_{0}^{\varepsilon}$ are closely related to a diffusion process that can be readily described. In particular, we find that the equation for computing $w_{\delta}(\omega, q)=\lim _{\varepsilon \rightarrow 0} \mathbb{E}\left\{R_{0}^{\varepsilon}(\omega+\right.$ $\left.\left.\varepsilon \frac{q}{2}\right) \overline{R_{0}^{\varepsilon}}\left(\omega-\varepsilon \frac{q}{2}\right)\right\}$ is very similar to its continuous counterpart.

To simplify the computation of $w_{\delta}(\omega, q)$, we analyze the case of a totally reflecting termination. This is consistent with the fact that we have Anderson localization. Indeed, since $\left|T_{J}^{\varepsilon}\right| \rightarrow 0$ when $J \rightarrow \infty$ it follows that $\left|R_{J}^{\varepsilon}\right| \rightarrow 1$ as $J \rightarrow \infty$.

Consequently, we set $R_{j}^{\varepsilon}(\omega)=\mathrm{e}^{\mathrm{i} \phi_{j}^{\varepsilon}(\omega)}$ where $\phi_{j}^{\varepsilon}(\cdot) \in \mathbb{R} / 2 \pi \mathbb{Z}$, and after its substitution in (B.1b) one gets that

$$
\phi_{j-1}^{\varepsilon}-\phi_{j}^{\varepsilon}=\frac{1}{\mathrm{i}} \log \left[\frac{1-\tilde{\sigma}_{j-1 / 2} \mathrm{e}^{-\mathrm{i}\left(\phi_{j}^{\varepsilon}-(2 j-1) \frac{\omega h}{\varepsilon}\right)}}{1-\tilde{\sigma}_{j-1 / 2} \mathrm{e}^{\mathrm{i}\left(\phi_{j}^{\varepsilon}-(2 j-1) \frac{\omega h}{\varepsilon}\right)}}\right] .
$$


Furthermore, we have to determine $w_{\delta}(\omega, q)=\lim _{\varepsilon \rightarrow 0} \mathbb{E}\left\{\mathrm{e}^{\mathrm{i}\left(\phi_{0}^{\varepsilon}\left(\omega+\varepsilon \frac{q}{2}\right)-\phi_{0}^{\varepsilon}\left(\omega-\varepsilon \frac{q}{2}\right)\right)}\right\}$ using the stationary solution of equation (B.4).

After introducing the new variables

$$
\psi_{j}^{\varepsilon, \pm}(\omega, q)=\phi_{j}^{\varepsilon}\left(\omega \pm \frac{\varepsilon}{2} q\right) \mp(j-1 / 2) \varepsilon q \delta
$$

one gets that $w_{\delta}(\omega, q)=\lim _{\varepsilon \rightarrow 0} \mathbb{E}\left\{\mathrm{e}^{\mathrm{i}\left(\psi_{0}^{\varepsilon,+}(\omega, q)-\psi_{0}^{\varepsilon,-}(\omega, q)\right)}\right\}$. And using the assumptions introduced above we obtain that

$$
\begin{aligned}
\psi_{j-1}^{\varepsilon, \pm}-\psi_{j}^{\varepsilon, \pm}=2 \sqrt{\varepsilon} \Delta \mu(j \delta) & \sin \left(\psi_{j}^{\varepsilon, \pm}-(2 j-1) \omega \delta\right) \pm \varepsilon q \delta \\
& +\varepsilon(\Delta \mu(j \delta))^{2} \sin \left(2\left(\psi_{j}^{\varepsilon, \pm}-(2 j-1) \omega \delta\right)\right)+O\left(\varepsilon^{3 / 2}\right)
\end{aligned}
$$

where $\Delta \mu(j \delta)=\mu(j \delta)-\mu((j-1) \delta)$.

These discrete equations can be recast in the form

$$
X_{j+1}^{\varepsilon}-X_{j}^{\varepsilon}=\sqrt{\varepsilon} F\left(j, X_{j}^{\varepsilon}\right) \xi_{j}+\varepsilon\left[\bar{G}\left(X_{j}^{\varepsilon}\right)+\tilde{G}\left(j, X_{j}^{\varepsilon}, \xi_{j}\right)\right]+O\left(\varepsilon^{3 / 2}\right)
$$

where $X_{j}^{\varepsilon} \in \mathbb{R}^{2},\left\{\xi_{j}\right\}$ is a random process and the functions $F, \bar{G}, \tilde{G}$ are $C^{\infty}$. Moreover, $\left\{\xi_{j}\right\}$ is a mean zero stationary mixing process with an exponentially decaying correlation function $R_{j}=\mathbb{E}\left\{\xi_{j} \xi_{0}\right\}$, the functions $F, \tilde{G}$ are almost-periodic on their first argument and the average $\langle\tilde{G}(j, X, \xi)\rangle_{j}=\lim _{N \rightarrow \infty}(N-j+1)^{-1} \sum_{k=j}^{N} \tilde{G}(k, X, \xi)=0$ for any fixed $X, \xi$.

Introducing the random process $X^{\varepsilon}(s)=X_{j}^{\varepsilon}$ on $[j \varepsilon,(j+1) \varepsilon)$ and slightly generalizing theorems 8 and 9 in [29, pp. 87-91] to consider the almost-periodic dependence, we get that when $\varepsilon \rightarrow 0$ the random function $\left\{X^{\varepsilon}(\cdot)\right\}$ weakly converges to a diffusion process $X(\cdot)$ with generator

$$
\begin{aligned}
\mathcal{A} f(x)=\bar{G}(x) f_{x}^{\prime}(x)+\frac{1}{2} R_{0}\left\langle F^{T}(j, x) f_{x x}^{\prime \prime}(x)\right. & F(j, x)\rangle_{j} \\
& +\left\langle\sum_{k=j+1}^{\infty} R_{j-k}\left[f_{x}^{\prime}(x) F(k, x)\right]_{x}^{\prime} F(j, x)\right\rangle_{j}
\end{aligned}
$$

where $f_{x}^{\prime}, f_{x x}^{\prime \prime}$ represent the gradient and the Hessian matrix of the function $f(x)$, respectively.

After a straightforward computation one gets that the limiting process $\psi(\cdot)$ associated with $\psi_{j}^{\varepsilon,+}-\psi_{j}^{\varepsilon,-}$ is a diffusion process with generator

$$
\mathcal{L} f(\psi)=2 q \delta f_{\psi}^{\prime}(\psi)+C_{\Delta \mu}(\delta, \omega)(1-\cos \psi) f_{\psi \psi}^{\prime \prime}(\psi)
$$

where

$$
C_{\Delta \mu}(\delta, \omega)=\sum_{j=0}^{\infty} R_{\Delta \mu}(j \delta) \cos (2 \omega j \delta),
$$

and $R_{\Delta \mu}(s)=\mathbb{E}\{\Delta \mu(s) \Delta \mu(0)\}$. Finally, one gets that $w_{\delta}(\omega, q)=\lim _{s \rightarrow \infty} V(s, \psi ; \omega, q)$ where $V(s, \psi ; \omega, q)$ is the solution of the initial value problem

$$
\begin{gathered}
\left(\partial_{s}-\mathcal{L}\right) V=0, \\
V(s=0, \psi)=\mathrm{e}^{\mathrm{i} \psi} .
\end{gathered}
$$


The equations (B.6) and (B.8) are similar to the ones obtained in [9, equations (4.14)-(4.16) on p. 592]. Consequently, $\Lambda_{\delta}(t, \omega)=(2 \pi)^{-1} \int \mathrm{e}^{-\mathrm{i} q t} w_{\delta}(\omega, q) \mathrm{d} q$ is explicitly expressed as

$$
\Lambda_{\delta}(t, \omega)=\frac{C_{\Delta \mu}(\delta, \omega)}{4 \delta\left(1+\frac{C_{\Delta \mu}(\delta, \omega)}{4 \delta} t\right)^{2}}
$$

where $C_{\Delta \mu}(\delta, \omega)$ is given by (B.7). After some simple algebra we get that $\frac{C_{\Delta \mu}(\delta, \omega)}{4 \delta}=$ $\Gamma_{\mu}^{\delta}(\omega)$.

\section{REFERENCES}

[1] D. Alfaro Vigo, J.-P. Fouque, J. Garnier and A. Nachbin, Robustness of time reversal for waves in time-dependent random media, Stoch. Processes Appl., 113, 289-313, 2004

[2] P. Anderson, Absence of diffusion in certain random lattices, Phys. Rev., 109, 1491-1505, 1958.

[3] M. Asch, W. Kohler, G. Papanicolaou, M. Postel and B. White, Frequency content of randomly scattered signals, SIAM Review, 33, 519-626, 1991.

[4] M. Asch, G. Papanicolaou, M. Postel, P. Sheng and B. White, Frequency content of randomly scattered signals. Part I, Wave Motion, 12, 429-450, 1990

[5] G. Bal, G. Papanicolaou and L. Ryzhik, Self-averaging in time reversal for the parabolic wave equation, Stochastics and Dynamics, 2, 507-531, 2002.

[6] G. Bal and L. Ryzhik, Time reversal for classical waves in random media, C. R. Acad. Sci. Paris, 333, 1041-1046, 2001.

[7] P. Blomgren, G. Papanicolaou and H. Zhao, Super-resolution in time-reversal acoustics, J. Acoust. Soc. Am., 111, 230-248, 2002.

[8] L. Brillouin, Wave Propagation in Periodic Structures, Dover Publications, New York, 2nd. ed., 2003.

[9] R. Burridge, G. Papanicolaou, P. Sheng and B. White, Probing a random medium with a pulse, SIAM J. Appl. Math., 49, 582-607, 1989.

[10] R. Burridge, G. Papanicolaou and B. White, Statistics for pulse reflection from a randomly layered medium, SIAM J. Appl. Math., 47, 146-168, 1987.

[11] J. Clouet and J.-P. Fouque, A time-reversal method for an acoustical pulse propagating in randomly layered media, Wave Motion, 25, 361-368, 1997.

[12] J. De Rosny, A. Tourin, A. Derode, B. van Tiggelen and M. Fink, Relation between time reversal refocusing and coherent backscattering in multiple scattering media: A diagrammatic approach, Phys. Rev. E, 70, 046601, 2004.

[13] A. Derode, P. Roux and M. Fink, Robust acoustic time reversal with high-order multiple scattering, Phys. Rev. Lett., 75, 4206-4209, 1995.

[14] A. Derode, A. Tourin and M. Fink, Random multiple scattering of ultrasound. ii: Is time reversal a self-averaging process? Phys. Rev. E, 64 , 6606, 2001.

[15] A. Derode, A. Tourin, J. D. Rosny, M. Tanter, S. You and M. Fink, Taking advantadge of multiple scattering to communicate with time reversal antennas, Phys. Rev. Lett., 90, $014301,2001$.

[16] F. Domínguez-Adame and V. Malyshev, A simple approach to Anderson localization in onedimensional disordered lattices, Am. J. Phys., 72, 226-230, 2004.

[17] B. Engquist, Computation of oscillatory solutions to hyperbolic differential equations, Nonlinear Hyperbolic Problems, C. Carasso, P.-A. Raviart, and D. Serre, eds., Lectures Notes in Mathematics, Springer-Verlag, New York, 1270, 10-22, 1987.

[18] B. Engquist and T. Hou, Particle method approximation of oscillatory solutions to hyperbolic differential equations, SIAM J. Numer. Anal., 26, 289-319, 1989.

[19] M. Fink, Time-reversal mirrors, J. Phys. D: Appl. Phys., 26, 1333-1350, 1993.

[20] M. Fink, Time-reversed acoustics, Sci. Am., November, 91-97, 1999.

[21] M. Fink and C. Prada, Eigenmodes of the time reversal operator: a solution to selective focusing in multiple-target media, Wave Motion, 20, 151-163, 1994.

[22] J.-P. Fouque, Acoustic pulses propagating in randomly layered media, Diffuse Waves in Complex Media, J.-P. Fouque, ed., Kluwer Academic Publishers, The Netherlands, 319-345, 1999.

[23] J.-P. Fouque and A. Nachbin, Time-reversed refocusing of surface water waves, Multiscale Model. Simul., 1, 609-629, 2003.

[24] S. Gredeskul and Y. Kivshar, Propagation and scattering of nonlinear waves in disordered systems, Phys. Repts., 216, 1-61, 1992. 
[25] M. Haider, K. Mehta and J.-P. Fouque, Time-reversal simulations for detection in randomly layered media, Waves in Random Media, 14, 185-198, 2004.

[26] M. Heinemann, A. Larranza, and K. Smith, Acoustic communications in an enclosure using single-channel time-reversal acoustics, Appl. Phys. Lett., 80, 694-696, 2002.

[27] A. Kim, P. Kyritsi, P. Blomgren and G. Papanicolaou, Spatial focusing and intersymbol interference in multiple input single output time reversal communication systems, submitted, 2005.

[28] W. Kuperman, W. Hodgkiss, H. Song, T. Akal, C. Ferla and D. Jackson, Phase conjugation in the ocean: experimental demonstration of an acoustic time-reversal mirror, J. Acoust. Soc. Am., 103, 25-40, 1997.

[29] H. Kushner, Approximation and Weak Convergence Methods for Random Processes, with Applications to Stochastic System Theory, Signal Processing, Optimization, and Control, MIT Press, Cambridge, Massachusetts, 1984.

[30] R. O'Doherty and N. Anstey, Reflections on amplitudes, Geophysical Prospecting, 19, 430-458, 1971.

[31] A. Pazy, Semigroups of Linear Operators and Applications to Partial Differential Equations, Appl. Math. Sci., Springer-Verlag, New York, 44, 1983.

[32] H. Schanz and U. Smilansky, Periodic-orbit theory of Anderson localization on graphs, Phys. Rev. Lett., 84, 1427, 2000

[33] K. Sølna, Focusing of time-reversed reflections, Waves Random Media, 12, 365-385, 2002.

[34] G. Strang, On the construction and comparison of difference schemes, SIAM J. Numer. Anal., 5, 506-517, 1968.

[35] J. Thomas and M. Fink, Ultrasonic beam focusing through tissue inhomogeneities with a time reversal mirror: Applications to transkull therapy, IEEE Trans. Ultrason. Ferroelectr. Freq. Control, 43, 1122-1129, 1996 